\title{
Coordinated Speed Oscillations in Schooling Killifish Enrich Social Communication
}

\author{
Daniel T. Swain ${ }^{1}$ - Iain D. Couzin $2,3,4$. \\ Naomi Ehrich Leonard ${ }^{1}$
}

\begin{abstract}
We examine the spatial dynamics of individuals in small schools of banded killifish (Fundulus diaphanus) that exhibit rhythmic, oscillating speed, typically with sustained, coordinated, out-of-phase speed oscillations as they move around a shallow water tank. We show that the relative motion among the fish yields a periodically time-varying network of social interactions that enriches visually driven social communication. The oscillations lead to the regular making and breaking of occlusions, which we term "switching." We show that the rate of convergence to consensus (biologically, the capacity for individuals in groups to achieve effective coordinated motion) governed by the switching outperforms static alternatives, and performs as well as the less practical case of every fish sensing every other fish. We show further that the oscillations in speed yield oscillations in relative bearing between fish over a range that includes the angles previously predicted to be optimal for a fish to detect changes in heading and speed of its neighbors. To investigate systematically, we derive and analyze a dynamic model of interacting agents that move with oscillatory speed. We show that coordinated circular motion of the school leads to systematic cycling of spatial ordering of agents and possibilities for enriched spatial density of measurements of the external environment. Our results highlight the potential benefits of dynamic communication topologies in collective animal behavior, and suggest new, useful control laws for the distributed coordination of mobile robotic networks.
\end{abstract}

$凶 \quad$ Naomi Ehrich Leonard naomi@princeton.edu

1 Mechanical and Aerospace Engineering, Princeton University, Princeton, NJ, USA

2 Ecology and Evolutionary Biology, Princeton University, Princeton, NJ, USA

3 Department of Collective Behaviour, Max Planck Institute for Ornithology, Konstanz, Germany

4 Department of Biology, University of Konstanz, Konstanz, Germany 
Keywords Network dynamics - Swarming - Collective animal behavior - Social information $\cdot$ Information transfer $\cdot$ Coordination

Mathematics Subject Classification $92 \mathrm{D} 50 \cdot 93 \mathrm{~A} 14 \cdot 93 \mathrm{~A} 30 \cdot 34 \mathrm{H} 05$

\section{Introduction}

Animals that move together in schools, flocks, and herds typically rely on social cues to maintain cohesion and to respond to changes in motion by other group members. How individuals obtain these social cues, however, is not well understood and depends on their sensing capabilities. For example, when social communication is dominated by sensing and sensing is restricted in range or direction, the group's spatial geometry can strongly influence information passing and thus group behavior. Many previous studies of mobile animal groups have attempted to explain observed behavior as resulting from average measures of group geometry such as average spacing and configuration (Spooner 1931; van Olst and Hunter 1970; Breder 1976; Partridge et al. 1980; Dill et al. 1997; Bajec and Heppner 2009). It has been shown from empirical data that such average measures of spatial distribution can remain steady even as individuals move continuously relative to one another [e.g., for juvenile blacksmith fish in Parrish and Turchin (1997)]. Conversely, even if average inter-neighbor distances remain fixed, each individual's set of neighbors may be constantly changing.

With static neighborhoods, information is passed from neighbor to neighbor. With dynamic neighborhoods, however, information may also be passed by individuals carrying information between neighborhoods. As a result, using average spatial structures in groups to understand information passing may be insufficient to explain how well information passes through these groups.

Models based on self-propelled particle dynamics have been successfully used to investigate emergence of collective motion from individual steering laws responsive to near neighbors (Vicsek et al. 1995; Gueron et al. 1996; Czirok et al. 1999; Couzin et al. 2002, 2005; Sumpter et al. 2008; Leonard et al. 2012). In these models, neighborhoods are dynamic, since although individuals move at a constant speed, they turn as a function of the relative direction (and/or position) of near neighbors. However, it has been shown that for schooling fish, such models cannot explain the way information propagates in groups, whereas sensory networks based explicitly on visual cues much better explain the mechanism of social communication (Strandburg-Peshkin et al. 2013). It remains a challenge to understand explicitly the role of the relative motion of individuals on information passing and collective behavior both when speeds are constant, and in scenarios where speeds fluctuate.

For visual information to pass directly from fish A to fish B, fish B must have line-of-sight visibility of fish A. Thus, if the fish in a school are distributed with constant relative positions, each fish will have a constant set of visible neighbors. Breder (1976) proposed that fish may form lattice-like structures in order to mutually satisfy separation preferences. Dill et al. (1997) proposed that fish may arrange themselves with preferred relative bearing angles in order to maximize sensitivity to various visual cues; these optimal bearing angles, predicted to be $35.3^{\circ}, 45.0^{\circ}, 63.4^{\circ}$, and $90.0^{\circ}$, also 
lead to a lattice structure. Lattice structures and other regular patterns create visual occlusions; a fish in such a distribution will have line of sight to near neighbors, but these near neighbors will occlude the visibility of further neighbors situated in the otherwise same line of sight.

In contrast, fish in a school that change their relative positions may have changing line-of-sight visibility and thus changing sets of visible neighbors as well as changing relative bearings. In this paper, we study the relative motion among individuals in small mobile schools of banded killifish (Fundulus diaphanus), which exhibit strikingly well-coordinated rhythmic, oscillating speeds. In particular, when one fish speeds up another slows down so that they move closer then farther from one another in a steady, periodic way. We quantify and evaluate the changing spatial geometry and the effect on social communication and collective behavior. To do so we analyze trajectories of individual fish that have been digitally reconstructed from overhead video of two-fish and three-fish schools, and we develop and apply a novel method based on the Hilbert transform that detects coordinated oscillations in the data and localizes them in time.

Killifish have available a variety of sensing modalities; in the present study we focus on social communication through visual cues. We examine how the quantified periodically changing relative positions remove visual occlusions during a portion of each oscillation cycle, and we estimate how this changing line-of-sight visibility implies a periodically time-varying sensing topology, i.e., the graph encoding which fish senses which other fish in the school switches periodically in time. While visual information is thought to be the primary sensory modality coordinating schooling in small freshwater fish (Hanke and Lauder 2006), occlusion would also inhibit other forms of social communication, such as via the "lateral line" of mechanosensitive cells that detects water movement (Chagnaud and Coombs 2014).

To quantitatively evaluate the effect of the switching sensing topology on information flow in the school, we compute the rate of convergence to consensus as predicted by consensus dynamics (Tsitsiklis 1984; Jadbabaie et al. 2003; Olfati-Saber and Murray 2003), a common model for how coordination in motion is achieved in animal groups (Vicsek et al. 1995; Czirok et al. 1999; Couzin et al. 2002, 2005). In the consensus model each individual modifies its decision variable according to the average of the value of each of its neighbor's variable. The rate of convergence to consensus refers to the rate at which the group converges to a common decision value (direction of travel).

We compare the rate of convergence to consensus associated with the switching sensing topology, estimated from the three-killifish data, to the rate of convergence to consensus computed in the case of no switching when the sensing topology remains fixed at one or the other of the sensing topologies. We draw specifically on work in Cao et al. (2008), Swain et al. (2008), in which the rate of convergence to consensus for time-varying sensing topologies was investigated. In Young et al. (2013), a related approach was used to evaluate performance, as measured by robustness of consensus to noise, of the social interaction network of starling flocks. The tradeoff between speed (rate of convergence) and accuracy (robustness to noise) in a decision-making network as a function of the network topology was studied in Srivastava and Leonard (2014).

The periodically changing relative positions among the killifish imply periodically changing relative bearings. This also has consequences for social communication, 
which we examine by comparing the estimated bearing angles to the optimal bearing angles predicted by Dill et al. (1997).

To enable a systematic examination of enrichment of social communication and collective behavior for a group of agents with coordinated speed oscillations, we derive an idealized continuous-time dynamic model motivated by the observed killifish behavior. These dynamics model a group of agents with oscillating speeds that use feedback to coordinate their headings and their speed oscillation phases. We explore straightline motion and circular motion, both of which are exhibited in the killifish data. We show how out-of-phase speed oscillations yield motion patterns with advantages that include enriched social communication as well as enriched spatial density of measurements of an external environmental field. The model and analysis can be applied to the study of collective animal behavior and to the design of mobile robotic networks.

The paper is organized as follows. In Sect. 2, we quantify coordinated speed oscillations in the schooling killifish experiments using our novel method for detecting coordinated oscillations and localizing them in time. In Sect. 3 we examine the changing spatial configurations and the switching in sensing for the three-fish schools. In Sect. 4 we evaluate the role of switching in sensing on convergence rate to consensus. In Sect. 5 we derive the dynamic model of agents with oscillating speed and study coordinated motion patterns. We discuss results in Sect. 6.

\section{Quantification of Coordinated Speed Oscillations}

In this section we quantify the remarkable speed oscillations exhibited in the tracked trajectories of schooling killifish swimming in a shallow water tank. We show that in the experimental data analyzed, these fish engage in consistent, rhythmic, coordinated speed oscillations.

We analyze the data collected in the experiments with two-fish and three-fish schools described in "Appendix 1." The fish in schools of four or more exhibited very similar behavior; we do not analyze their behavior here. Banded killifish naturally school in very shallow water, including the depth employed in our experiments (Krause et al. 2000). Furthermore, since they swim along the bottom of the water body, feeding as they move, they tend to occupy the lower few cm even in deeper water.

In these experiments, the killifish, which were between 3 and $4 \mathrm{~cm}$ long, swam in 2.5 -cm-deep water in a $155-\mathrm{cm}$ square tank and were recorded from a video camera above the tank at 31.2 frames per second. The trajectories of the fish were tracked from the video yielding the horizontal position of each fish $\mathbf{r}(t)=(x(t), y(t))$ at the time of every video frame $t$. There are 15 groups of fish used for the analysis of speed oscillations in two-fish schools and 11 groups of fish used for the analysis of speed oscillations and the analysis of configurations of three-fish schools. These data were sorted into time segments, with a small number of segments being rejected in the case fish were idle or not visible in the video; see "Appendix 1" for details. The segmenting and the total length of time of the data analyzed for the different analyses is summarized in Table 1.

The tracked horizontal position $\mathbf{r}(t)$ over time of each fish was used to estimate the velocity $\mathbf{v}(t)$ of each fish at each time $t$ during each segment. From $\mathbf{v}(t)$, the 
Table 1 Summary of number of time segments of the video and total time of segments analyzed for two-fish and three-fish speed oscillations and for three-fish configurations

\begin{tabular}{lllcc}
\hline $\begin{array}{l}\text { Analysis } \\
\text { type }\end{array}$ & School size & $\begin{array}{l}\text { Number of } \\
\text { groups }\end{array}$ & $\begin{array}{l}\text { Number of } \\
\text { segments }\end{array}$ & $\begin{array}{l}\text { Total time } \\
(\mathrm{min})\end{array}$ \\
\hline Speed oscillations & 2 & 15 & 130 & 22.39 \\
Speed oscillations & 3 & 11 & 63 & 15.21 \\
Configurations & 3 & 11 & 246 & 18.11 \\
\hline
\end{tabular}

speed of the fish is calculated as $s(t)=\|\mathbf{v}(t)\|$ and its heading as $\theta(t)=\angle\{\mathbf{v}(t)\}$, where $\angle\{\mathbf{v}(t)\}$ is the angle that $\mathbf{v}(t)$ forms with the $x$-axis. When speed oscillations are present, they are oscillations about the nominal speed, $\bar{s}(t)$. The nominal speed is the "average" speed of the fish; this average slowly varies over time, so we compute it using a moving-window average as described in "Appendix 2." In our method for detecting speed oscillations we also use the speed variation $\delta s(t)$ of each fish, which is defined as the speed minus the nominal speed:

$$
\delta s(t)=s(t)-\bar{s}(t) .
$$

\subsection{Method for Detecting Coordinated Speed Oscillations}

In order to quantitatively detect coordinated speed oscillations from the data with a high degree of localization both in time and frequency, we developed an algorithm based on the Hilbert transform. For a thorough discussion of the estimation of instantaneous phase and, in particular, the Hilbert transform method, see Boashash (1992). Our algorithm locates subsequences of the speed variation sequence $\delta s(t)$, as defined in (1), that fit well to a pure sinusoid and then estimates the corresponding frequency, amplitude, and phase. We can isolate subsequences during which two or more fish engage in coordinated speed oscillations: When two or more fish in a subsequence exhibit sinusoidal speed variation with approximately the same frequency, then we refer to them as coordinated. The coordination patterns can be characterized by the relative phasing of the speed oscillations.

Our algorithm leverages properties of the analytic signal of a sequence, which is defined as the complex quantity with imaginary part equal to the Hilbert transform of the sequence and real part equal to the original sequence. For example, the analytic signal, $\delta \hat{s}(t)$, of the speed variation is

$$
\delta \hat{s}(t)=\delta s(t)+\sqrt{-1} H(\delta s(t)),
$$

where $H(\delta s(t))$ is the Hilbert transform of $\delta s(t)$. The analytic signal is estimated using MATLAB's hilbert command.

It can be shown for nearly sinusoidal signals that the angular argument of the analytic signal gives an estimate of instantaneous phase and that the magnitude of the analytic signal gives an estimate of instantaneous amplitude. For purely sinusoidal signals, the instantaneous phase grows linearly in time with the constant slope equal to 

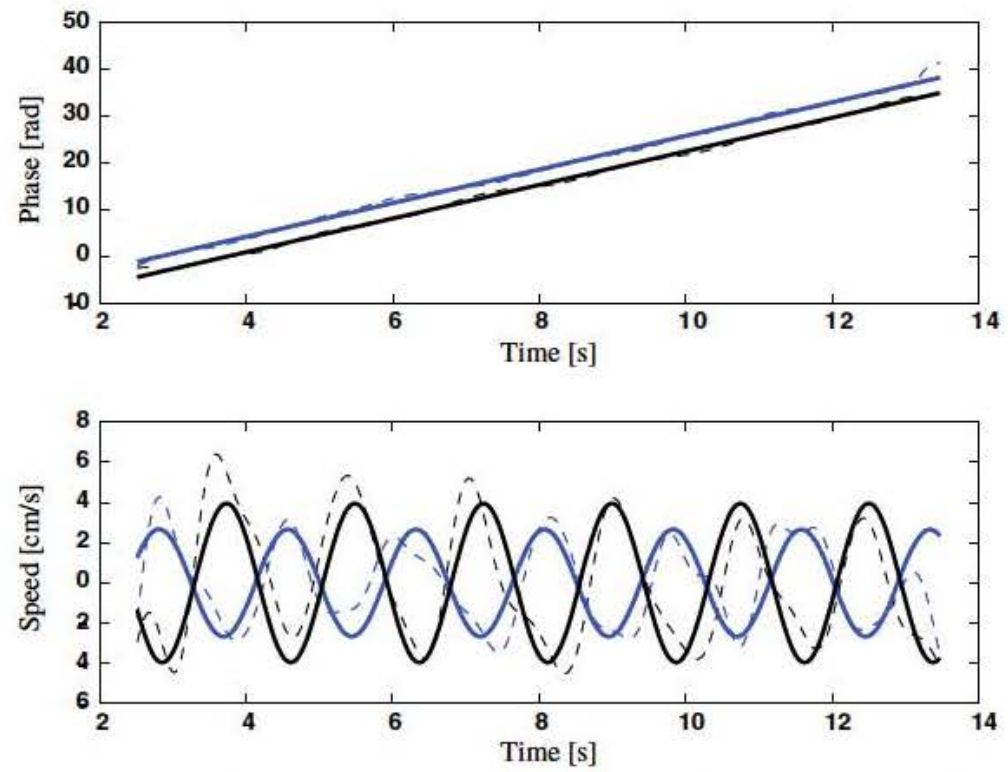

Fig. 1 The speed variation (bottom) and instantaneous phase (top) as a function of time for each of the fish in an example segment of the two-fish data. The dashed curves correspond to the data, and the solid curves correspond to the fit. The slope of the lines fitted to the instantaneous phase provides an estimate of the frequency of oscillation. In this time segment the fish are estimated to be oscillating at the same frequency with a phase difference of approximately $180^{\circ}$

the frequency. Therefore, we look for subsequences in the data over which the angular argument of the analytic signal (i.e., the instantaneous phase estimate) fits well to a line. The slope of the fitted line gives an estimate of the frequency of oscillation.

We use a piecewise linear fitting algorithm to fit the instantaneous phase of the speed variation for each fish, plotted as a function of time, to a series of line segments. Each line segment represents a subsequence over which that fish may be oscillating its speed. The slope of each fitted line segment gives an estimate of the corresponding frequency of oscillation and the intercept on the instantaneous phase axis gives an estimate of the phase offset. The variance of the error from the best-fit line is used to measure fit quality, and a minimum allowable segment time length is enforced. For analysis of individual fish in the three-fish data, we consider only those subsequences of oscillating speed for which the average amplitude of oscillation is at least $25 \%$ of the nominal speed.

The method is illustrated in Fig. 1 for an example segment of two-fish data. In the bottom panel the speed variation of each of the fish is plotted as a function of time (blue and black dashed lines) and in the top panel the corresponding estimate of the instantaneous phase is plotted for each fish as a function of time (blue and black dashed lines). The solid fitted line segments in the top plot are parallel, which implies that the fish have speed oscillations of approximately the same frequency. The phase difference of the speed oscillations is estimated to be approximately $180^{\circ}$. The fitted (sinusoidal) speed variation of each of the fish is shown as a solid curve in the bottom plot. The out-of-phase speed oscillations can be observed. 
To identify segments of coordinated speed oscillations, we look for subsequences over which a pair of fish exhibits speed oscillations with similar frequencies, as in the case plotted in Fig. 1. The common oscillation frequency for each coordinated oscillation subsequence is calculated by finding the slope of the least-squares fit line for the instantaneous phase estimates of both fish simultaneously. The speed oscillations are taken to be coordinated if the percent difference in frequencies between two fish is below a maximum threshold. A minimum allowable segment time length is enforced. To estimate the relative phasing of a pair of fish with coordinated speed oscillations, we take the difference of the estimated phase offset for each fish.

For the analysis of configurations in the three-fish data (Sect. 3), we isolate segments over which pairs of fish exhibit strongly out-of-phase speed oscillations using an alternative approach. This approach identifies a pair of fish with large relative phase in speed oscillations (i.e., close to out-of-phase oscillations) by directly estimating the phase of the relative speed variation for the pair of fish $i$ and $j: \delta s_{i j}(t)=\delta s_{i}(t)-\delta s_{j}(t)$. The speed oscillation detection algorithm based on the Hilbert transform, as described above, is applied to $\delta s_{i j}(t)$. The instantaneous phase estimate of $\delta s_{i j}(t)$ is calculated, and subsequences of sufficient length for which the instantaneous phase fit sufficiently well to a line are reported as oscillatory subsequences. The oscillating speeds are then determined to be sufficiently out of phase if the average estimated amplitude (average magnitude of the analytic signal) of $\delta s_{i j}(t)$ is sufficiently large.

\subsection{Results on Strongly Coordinated Speed Oscillations}

The methods of Sect. 2.1 were applied to both the two-fish and three-fish trajectory data to identify and quantify speed oscillations. Figures $2 b$ and $3 b$ show sample speed profiles for two-fish and three-fish schools, respectively. The oscillations are sustained over the $12 \mathrm{~s}$ segment shown, and there is strong coordination of oscillation frequency and strong anti-synchronization of phases in both the two-fish school and in pairs of fish in the three-fish school.

In Fig. $2 \mathrm{~b}$ the phase difference of the oscillations can be observed to be consistently close to $180^{\circ}$, i.e., when one fish reaches a peak in speed, the other fish reaches a trough in speed. This leads to regular oscillations in relative positions of the two fish as can be observed in Fig. 2a. Figure 2a shows the trajectories of the two fish (moving up and to the left) in the experimental arena and four snapshots of the fish corresponding to peaks and troughs in the oscillating distance curve, shown in black in Fig. 2b. At the time of the earliest snapshot the two fish are side-by-side: The fish in green is accelerating, while the fish in blue is decelerating and the relative distance is at a trough. At the time of the second snapshot the fish in green has pulled ahead but is decelerating while the fish in blue is accelerating and the relative distance is at a peak. The pattern repeats itself: In the third snapshot the two fish are side-by-side again, the fish in blue having caught up, and in the fourth snapshot the fish in green is again ahead. We note that this changing configuration is highly representative of the behavior observed in the two-fish schooling experiments.

In Fig. $3 \mathrm{~b}$ the phase difference of the oscillations between the fish in red and each of the other two fish can be observed to be close to $180^{\circ}$, consistently in the first two seconds of the segment. This leads to regular oscillations in relative positions of the 


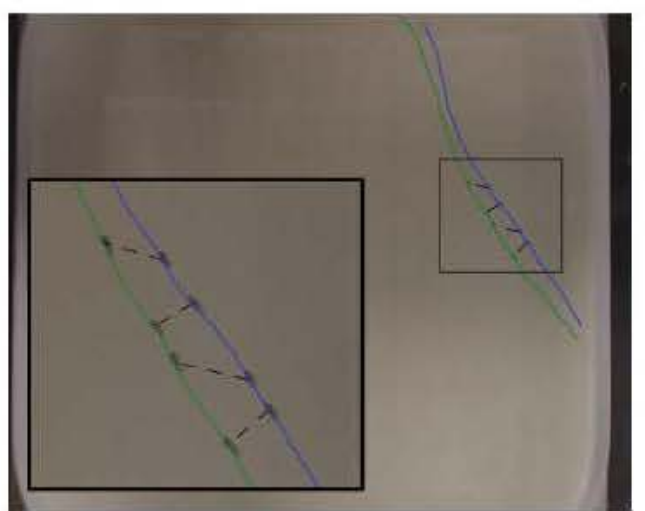

(a)

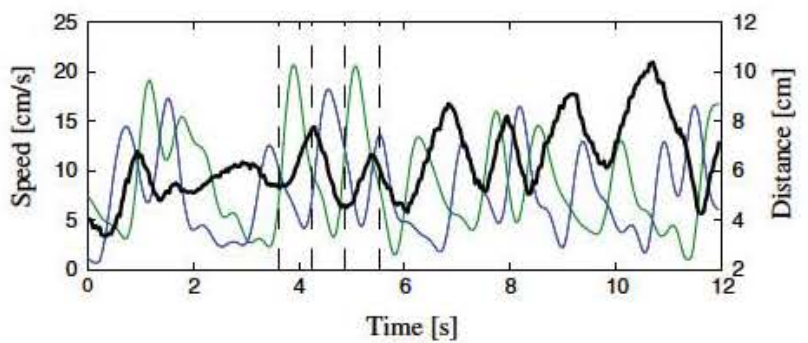

(b)

Fig. 2 Relative position oscillations of a two-fish school. a The trajectories (blue and green curves moving $u p$ and left) of the two fish in the experimental arena over a $12 \mathrm{~s}$ segment. Four snapshots of the two fish (each snapshot shows the fish connected by a dashed black line) are superimposed at times indicated by dashed, black vertical lines in b. Inset A close-up of the boxed region. In the earliest snapshot the two fish move side-by-side, in the second snapshot the fish in green is ahead, in the third snapshot the pair is side-by-side again, and in the fourth snapshot the fish in green is ahead. b The distance between the two fish (black curve) and the speed of each of the two fish (blue and green curves)

three fish as can be observed in Fig. 3a. Figure 3a shows the trajectories of the three fish (moving down and to the right) in the experimental arena and four snapshots of the fish corresponding to peaks and troughs in the oscillating distance curves, shown in black in Fig. 3b. At the time of the earliest snapshot the fish are in a "V"-shaped formation: The fish in red is lagging both the fish in green and the fish in blue, but it is accelerating, while the fish in green and blue are decelerating. The fish in green and blue stay in phase, and their relative position does not change much. The distance between the fish in red and both the fish in blue and in green oscillates. In the second snapshot the fish in red catches up to the other two fish; it is likely that the fish in green creates an occlusion so there is no line-of-sight visibility between the fish in red and the fish in blue. The pattern repeats itself: The fish in red lags again in the third snapshot, breaking the occlusion, but catches up in the fourth snapshot, leading again to an occlusion.

In Fig. 4 the phase difference of the oscillations between the fish in green and each of the other two fish can be observed to be close to $180^{\circ}$, consistently from 2 to $4 \mathrm{~s}$ of the segment. This leads to regular oscillations in relative positions of the three fish as 


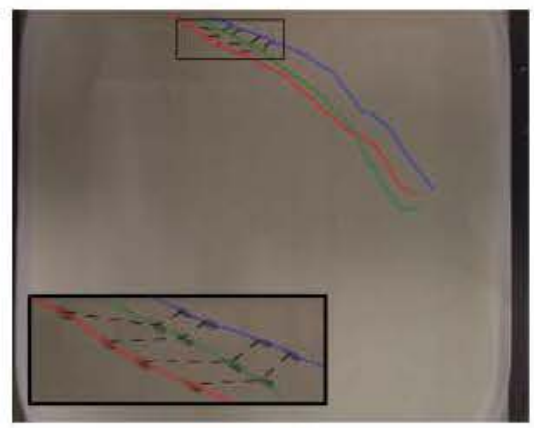

(a)

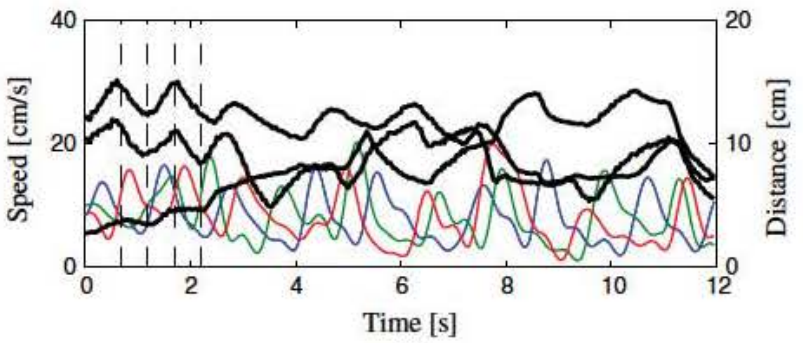

(b)

Fig. 3 Relative position oscillations of a three-fish school. a The trajectories (blue, green and red curves moving down and right) of the three fish in the experimental arena over a $12 \mathrm{~s} \mathrm{segment.} \mathrm{Four} \mathrm{snapshots} \mathrm{of}$ the three fish (each snapshot shows the fish connected by dashed black lines) are superimposed at times indicated by dashed, black vertical lines in b. Inset A close-up of the boxed region. In the earliest snapshot the fish in red lags the other two, in the second snapshot the fish in red catches up, repeating in the third and fourth snapshots. b The distance between each pair of fish (black curves) and the speed of each fish (blue, green and red curves)

can be observed in Fig. 4a. Figure 4a shows the trajectories of the three fish as in Fig. 3a and four snapshots of the fish corresponding to peaks and troughs in the oscillating distance curves, shown in black in Fig. 4 b. At the time of the earliest snapshot the fish are in a "V"-shaped formation: The fish in green creates an occlusion for line-of-site visibility between the fish in red and the fish in blue, but the fish in green is accelerating and both the fish in red and the fish in blue are decelerating. In the second snapshot the fish in green moves well ahead of the other two fish, breaking the occlusion. The pattern repeats itself: The fish in green decelerates and creates the occlusion again in the third snapshot, but moves well ahead in the fourth snapshot, again breaking the occlusion.

The results of the analysis of all of the two-fish and three-fish data used for speed oscillation analysis are presented in Table 2 . The results for the two-fish and threefish data show that killifish engage in regular, coordinated, speed oscillations with significant phase differences between pairs of fish leading to regular relative motion patterns.

As presented in Table 2, the fish are estimated to be oscillating their speed most of the time: Individually, they oscillate $92.8 \%$ of the time in the two-fish schools and $83.3 \%$ of the time in the three-fish schools. In the case of the two-fish schools, the 


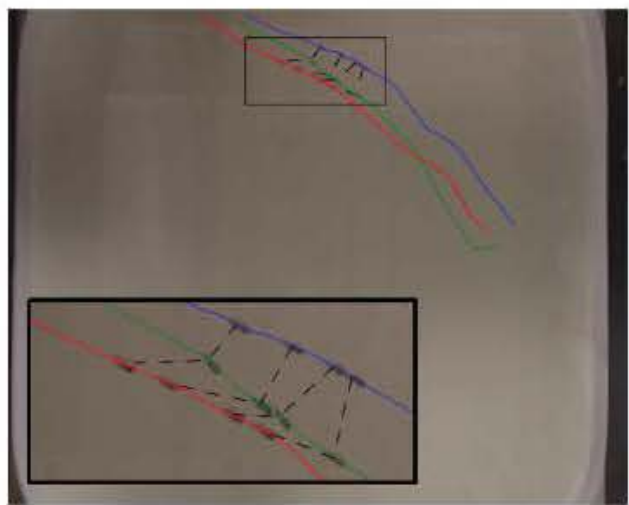

(a)

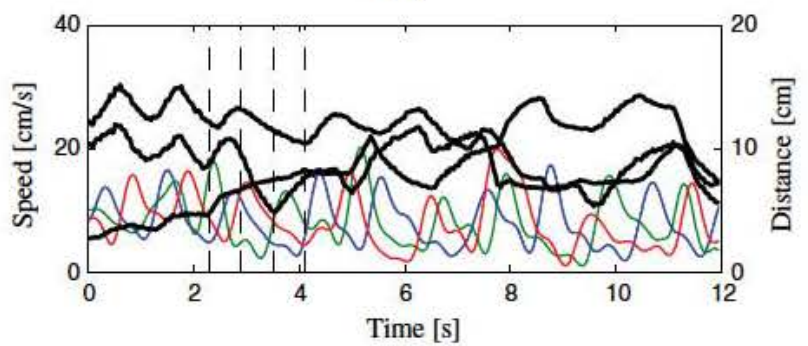

(b)

Fig. 4 Relative position oscillations of a three-fish school. The presentation is as in Fig. 3 except that the four snapshots are taken later in time. In the earliest snapshot the fish in green creates an occlusion between the other two fish, in the second snapshot the green fish moves ahead breaking the occlusion, in the third snapshot the fish in green creates the occlusion again, and in the fourth snapshot the fish in green moves ahead again

fish are not only oscillating but also oscillating at the same (coordinated) frequency as much as $76.9 \%$ of the time. It is further noteworthy that the fish across the two-fish and three-fish schools oscillate their speeds with the same consistent frequency $(0.75$ $\mathrm{Hz})$ and amplitude $(4 \mathrm{~cm} / \mathrm{s})$ about the same nominal speed $(8 \mathrm{~cm} / \mathrm{s})$. From this we can observe that the oscillations are significant in amplitude, at approximately $50 \%$ of the nominal speed. Further, since the tail beat frequency of these fish is greater than $1 \mathrm{~Hz}$, the speed oscillations with frequency $0.75 \mathrm{~Hz}$ are clearly distinguished from tail beat.

Because the difference in estimated phases of speed oscillations for a coordinating pair of fish will be susceptible to noise, we apply a metric called the mean sign product (MSP), which is less sensitive to amplitude fluctuations and noise in the speed variation signals. The MSP is the sample covariance of the signs of the speed variations of fish $i$ and fish $j$ over the sequence of time samples, as derived in "Appendix 3." To get a measure of coordination of speed oscillation phases among the fish, the MSP was calculated for nearest neighbor pairs during coordinated oscillations in both the two-fish and three-fish schools. As presented in Table 2 the MSP for the two-fish schools is on average -0.15 . This implies that the speed oscillations tend toward anti-synchronized phasing, consistent with observations from the data (as illustrated in Fig. 2). The MSP for near neighbors in the three-fish schools is on average 0.0. 
Table 2 Summary of analysis of speed oscillations in two-fish and three-fish schooling data

\begin{tabular}{llllllll}
\hline & School size & $\begin{array}{l}\text { \% of time } \\
\text { oscillating }\end{array}$ & $\begin{array}{l}\text { Frequency } \\
(\mathrm{Hz})\end{array}$ & $\begin{array}{l}\text { Amplitude } \\
(\mathrm{cm} / \mathrm{s})\end{array}$ & $\begin{array}{l}\text { Nominal } \\
(\mathrm{cm} / \mathrm{s})\end{array}$ & $\begin{array}{l}\text { MSP for } \\
\text { pairs }\end{array}$ \\
\hline Individuals & 2 & 92.8 & $0.75 \pm 0.24$ & $4.3 \pm 1.1$ & $8.0 \pm 2.1$ & N/A \\
Pairs & 2 & 76.9 & $0.74 \pm 0.16$ & $4.2 \pm 0.84$ & $8.1 \pm 1.7$ & $-0.15 \pm 0.27$ \\
Individuals & 3 & 83.3 & $0.76 \pm 0.25$ & $4.1 \pm 2.6$ & $8.3 \pm 2.7$ & $0.0 \pm 0.27$ \\
\hline
\end{tabular}

The analysis of pairs refers to the case in which both fish oscillate with the same frequency at the same time. The MSP is calculated for the three-fish data for segments when near neighbors oscillate with the same frequency at the same time

This suggests the possibility of a uniform distribution of relative phases in these pairs. However, observations from the data suggest that it is more likely the result of near neighbors sometimes being anti-synchronized and sometimes being synchronized. This is the case in Fig. 3 where the fish in green is synchronized with its near neighbor in blue and anti-synchronized with its near neighbor in red. The fish in blue and the fish in red are second neighbors and sustain anti-synchronized oscillations. This type of relative phasing leads to the periodically changing spatial configuration and switching sensing topology described in Sect. 3.1, as does the example in Fig. 4 where near neighbors are anti-sychronized and second neighbors are synchronized.

\section{Switching Configurations and Social Communication}

In this section we examine the relative motion of the three-fish data and identify the observed periodically time-varying configurations. We show two striking results on the cycling about these configurations in terms of social communication. First, we find the two most common configurations to be a Vformation and a diagonalformation, and we show how the cycling about these common configurations regularly makes and breaks occlusions of line-of-sight visibility between pairs of fish. Then, we show how the periodic relative motion facilitates periodic cycling between different relative bearings that have been predicted by Dill et al. (1997) to be optimal for social communication.

\subsection{Measuring Three-Fish Configurations}

We define the configurations of three-fish schools in terms of relative bearing from one fish identified as the central fish. The central fish, with position at time $t$ denoted $r_{c}(t)$ and direction of motion at time $t$ denoted $\theta_{c}(t)$, is defined as the fish closest to the centroid $\overline{\mathbf{r}}(t)$ of the school:

$$
\overline{\mathbf{r}}(t)=\frac{1}{N} \sum_{i=1}^{N} \mathbf{r}_{i}(t)
$$

The relative bearing, $\beta_{i}(t)$, of fish $i$ with respect to the central fish is estimated as

$$
\beta_{i}(t)=L\left\{\mathbf{r}_{i}(t)-\mathbf{r}_{c}(t)\right\}-\theta_{c}(t), i=1,2
$$




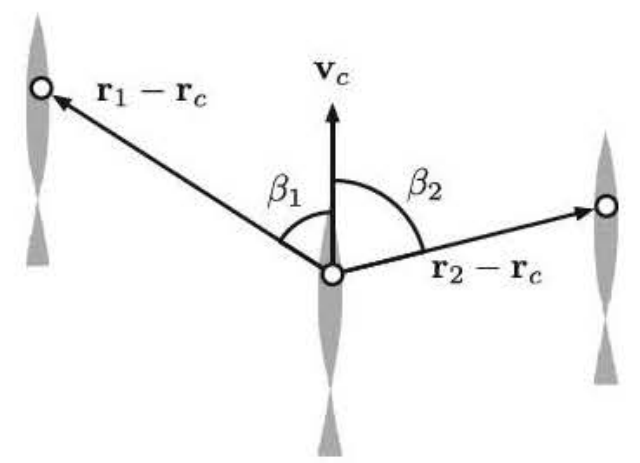

Fig. 5 Relative bearings $\beta_{1}$ and $\beta_{2}$, of fish 1 and 2, with respect to the central (middle) fish are used to quantify configurations. The relative bearing to fish $i$ is the angle of the relative position $\mathbf{r}_{i}-\mathbf{r}_{c}$ (from the centroid of the central fish to the centroid of fish $i$ ) relative to the velocity $\mathbf{v}_{c}$ of the central fish. The two angles are labeled such that $\beta_{1}>\beta_{2}$, and hence, $\beta_{1}\left(\beta_{2}\right)$ is always the angle to the leftmost (rightmost) neighbor of the central fish. In the figure, $\beta_{1}>0$ and $\beta_{2}<0$

and calculated in the range of $-180^{\circ}$ to $180^{\circ}$. See Fig. 5 for an illustration.

This pair of relative bearings gives a quantitative measurement of the spatial configuration of the school. The identities of the neighbors are ordered so that $\beta_{1}>\beta_{2}$. That is, $\beta_{1}$ is the relative bearing from the central fish to its leftmost neighbor and $\beta_{2}$ is the relative bearing from the central fish to its rightmost neighbor (both with respect to the direction of motion of the central fish); see Fig. 5.

\subsection{Results on Switching Configurations and Social Communication}

Figure 6 shows a two-dimensional histogram of the frequency of relative bearings $\beta_{1}$ and $\beta_{2}$ for the three-fish groups as defined above in Sect. 3.1. The histogram is plotted as a heat map ranging from blue, for low histogram counts, to red, for high histogram counts. The histogram was computed by calculating $\beta_{1}$ and $\beta_{2}$ at each time instant as described above and binning the values in $15^{\circ}$ by $15^{\circ}$ bins. Red regions in the histogram correspond to the most common configurations of the three-fish groups.

The regions corresponding to the two most common configurations are circled in Fig. 6, with arrows connecting these regions of the histogram to illustrations of the corresponding spatial configurations. There are three fish in each configuration, and each configuration is drawn at three separate time steps to demonstrate how the configuration changes as a result of relative motion in each of the two cases. The middle three-fish configuration in each of the illustrations shows the nominal configuration corresponding to the histogram peaks. In the nominal configuration in each of the two cases shown, there is an occlusion such that the leftmost and the rightmost fish cannot see each other: Their line of sight to each other is blocked by the central fish. In each case shown, the left and right configurations show changes from the nominal configuration that result from relative motion due to coordinated speed oscillations when the two outer (leftmost and rightmost) fish are anti-synchronized with the middle fish (as in the example shown in Fig. 4). That is, the fish move relative to one another 


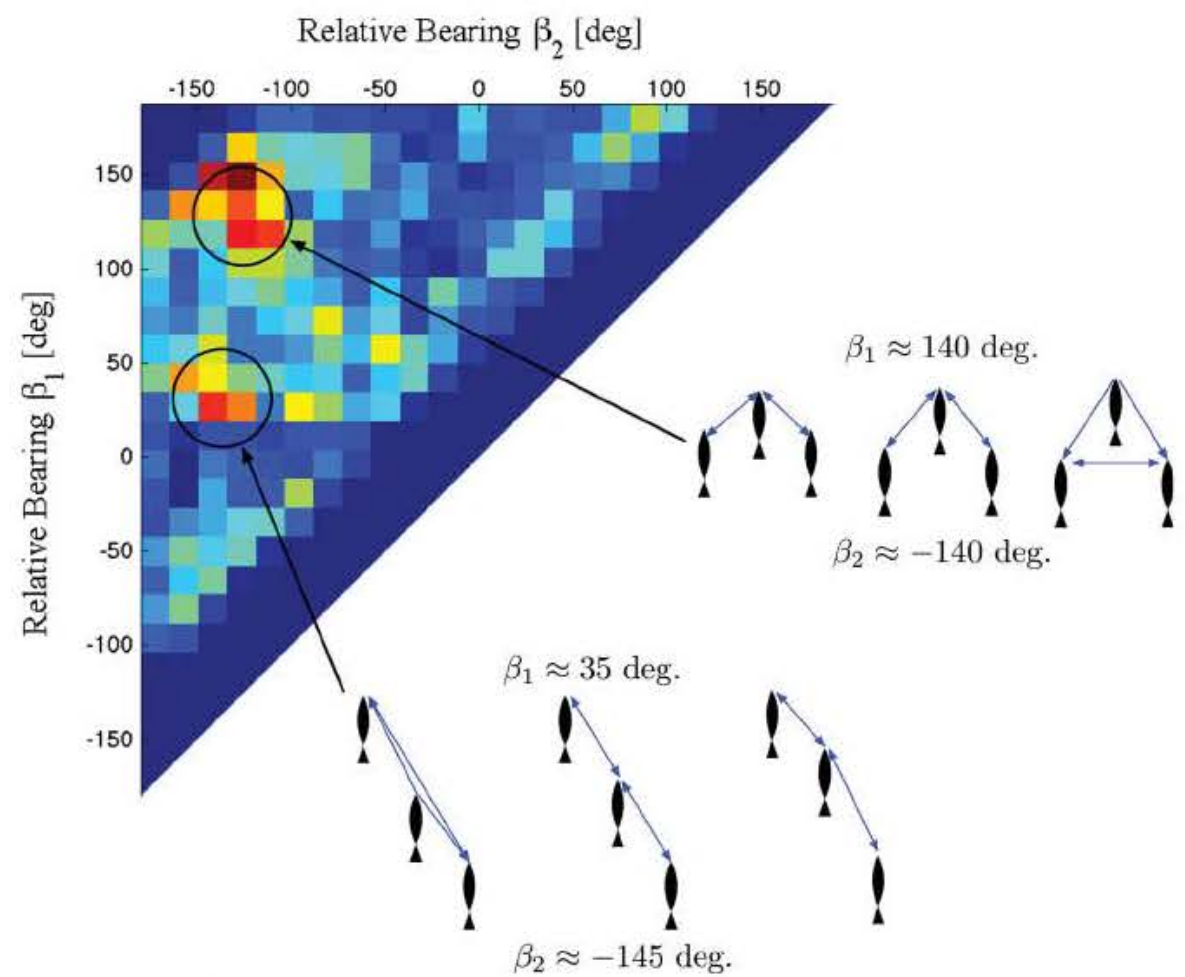

Fig. 6 (Top left) Histogram of configurations of groups of three killifish. $\beta_{1}\left(\beta_{2}\right)$ is the relative bearing from the central fish to its leftmost (rightmost) neighbor (see Fig. 5). Results are calculated at each time step and binned into $15^{\circ}$ by $15^{\circ}$ bins, producing a two-dimensional histogram. The histogram is plotted as a heat map ranging from blue, for low histogram counts, to red, for high histogram counts. Red regions therefore correspond to the most common configurations. (Bottom right) Representations of the two most common configurations (middle in each case), with configuration changes (left and right) due to relative motion resulting from speed oscillations. Arrows indicate which fish are estimated to be sensing which other fish

as their speeds oscillate and the configurations cycle between the left, nominal, and right configurations shown. A very similar kind of oscillation in configuration (notably with the same consequences for sensing topology) occurs when one of the outer fish is synchronized with the middle fish and the other outer fish is anti-synchronized with both of these fish (as in the example shown in Fig. 3). The relative motion in all of these cases contributes to the spread of the peaks in the histogram of Fig. 6 over several neighboring bins.

The most common case, referred to as the $V$ formation, is the one shown with nominal configuration given by $\left(\beta_{1}, \beta_{2}\right) \approx\left(140^{\circ},-140^{\circ}\right)$. This corresponds to a "V"-shaped formation with the central fish at the front of the group. The average distance between the middle and either outer fish is $7.1 \pm 2.7 \mathrm{~cm}$. Relative motion regularly produces periods of time for which the central fish occludes line-of-sight visibility between the two outer fish; see Figs. 3 and 4. During one part of the cycle 
(the configuration on the right of the nominal in the illustration), this occlusion is broken.

The second most common case, referred to as the diagonal formation, is the one shown with nominal configuration given by $\left(\beta_{1}, \beta_{2}\right) \approx\left(35^{\circ},-145^{\circ}\right)$. This corresponds to an angled line formation with the leftmost fish at the front of the group and each of the other two fish having its neighbor nominally at a $35^{\circ}$ relative bearing. Relative motion produces periods of time for which the central fish occludes the line-of-sight visibility between the two outer fish. During one part of the cycle (the configuration on the left of the nominal in the illustration), this occlusion is broken. Note that the left/right symmetric formation-i.e., with the rightmost fish at the front-is not present in the histogram. This could be due to the tendency of the fish to swim in a counter-clockwise direction around the tank, with the innermost fish (farthest from the wall) at the front of the group.

It is striking that the periodic relative motion observed facilitates periodic cycling between different relative bearings that have been predicted by Dill et al. (1997) to be optimal for social communication. The angles predicted by Dill et al. (1997) provide the optimal relative bearing (i.e., $\beta_{1}$ and $\beta_{2}$ in Fig. 5) from a focal fish to a neighbor fish with respect to the focal fish's ability to detect changes in the heading or speed of the neighbor. Dill et al. considered three visual cues: the angular velocity of the retina angle corresponding to the image of the eye of the neighbor fish, the loom, ${ }^{1}$ and the time to collision. ${ }^{2}$ Six optimal relative bearings were calculated by finding the relative bearing that maximizes the sensitivity of each visual cue (angular velocity, loom, or time to collision) to each behavioral change (turning or speed change). Four distinct optimal bearing angles emerge as follows: $35.3^{\circ}$ (for time to collision with respect to both speed and heading changes), $45.0^{\circ}$ (for angular velocity with respect to a heading change), $63.4^{\circ}$ (for loom with respect to a speed change), and $90.0^{\circ}$ (for angular velocity with respect to a speed change and loom with respect to a heading change). It was suggested that these values may predict the average relative bearings observed in animal groups. The results in Fig. 6 show that the speed oscillations of the killifish cause the relative bearing between fish to oscillate over a range that includes $35.3^{\circ}, 45.0^{\circ}$, and $63.4^{\circ}$, i.e., three of the four predicted optimal angles.

\section{Switching Configurations and Convergence to Consensus}

In this section we explore further the analysis in Sect. 3.2 of switching configurations that make and break visual occlusions and provide a quantitative measure of the role of the switching on the rate of convergence among the fish to a consensus. We show remarkably that the switching about the two most common configurations improves this convergence rate. First we estimate, for the three-fish data, the periodic switching sensing topology that results from the repeated making and breaking of occlusions. We then examine consensus dynamics for a group using this switching sensing topology

\footnotetext{
1 The loom, $\Lambda$, is the time rate of change of the solid angle, $\alpha$, subtended on the focal fish's eye by the eye of the neighbor.

2 The time to collision is equal to $2 \frac{\alpha}{\Lambda}$.
} 
and compute the rate at which these dynamics converge to a consensus. We show that the rate of convergence to consensus in the case of switching is faster than in the case of no switching.

\subsection{Model of Social Communication}

We model social communication of information by means of a time-varying graph $\mathcal{G}(t)$ that encodes the time-varying visual sensing topology, i.e., who is getting visual cues from whom as a function of time. A graph $\mathcal{G}(t)$ is defined by a node set $\mathcal{V}=\{1, \ldots, N\}$ and an edge set $\mathcal{E}(t) \subseteq \mathcal{V} \times \mathcal{V}$. Fish $i$ is identified with node $i$, and the number of nodes $N$ is the number of fish in the school. If at time $t$ fish $i$ is getting visual information from fish $j$, then $(i, j) \in \mathcal{E}(t)$, i.e., there is an edge in the graph from node $i$ to node $j$. The neighborhood $\mathcal{N}_{i}(t)$ of node $i$ at time $t$ is the subset of $\mathcal{V}$ such that $j \in \mathcal{N}_{i}(t) \subseteq \mathcal{V}$ if $(i, j) \in \mathcal{E}(t)$.

To construct the sensing topology for the three-fish groups that exhibit periodically time-varying occlusions as a result of coordinated speed oscillations, we make the following conservative assumptions about which fish get visual information from which: (1) Fish $i$ has fish $j$ as a neighbor only if fish $i$ has line-of-sight visibility of fish $j$. (2) Each fish has as many as two neighbors only if one is left and one is right of its heading direction. When both are visible on the same side, then only the most recently unoccluded fish is taken as its neighbor. (3) Fish $i$ applies weight $1 /\left|\mathcal{N}_{i}\right|$ to the information it gets from each of its neighbors $j \in \mathcal{N}_{i}$. So, if a fish has two neighbors it splits its attention equally between the information it gets from each. Given these assumptions, the Laplacian matrix $L(t)$ associated with $\mathcal{G}(t)$ is defined as

$$
L_{i j}(t)= \begin{cases}-\frac{1}{\left|\mathcal{N}_{i}(t)\right|}, & i \neq j, \quad j \in \mathcal{N}_{i}(t) \\ 0, & i \neq j, \quad j \notin \mathcal{N}_{i}(t) \\ 1, & i=j,\left|\mathcal{N}_{i}(t)\right| \neq 0 \\ 0 & i=j, \quad\left|\mathcal{N}_{i}(t)\right|=0\end{cases}
$$

Periodically time-varying occlusions imply a periodically time-varying graph $\mathcal{G}(t)=\mathcal{G}(t+T)$ with period $T$. As will be shown, $\mathcal{G}(t)$ estimated from the three-fish data switches in one period between two constant graphs $\mathcal{G}_{A}$ and $\mathcal{G}_{B}$. Let $p \in[0,1]$ be the duty cycle. Then,

$$
\mathcal{G}(t)=\left\{\begin{array}{l}
\mathcal{G}_{A}, t \in n T+[0,(1-p) T) \\
\mathcal{G}_{B}, t \in n T+[(1-p) T, T),
\end{array}\right.
$$

where $n=\left\lfloor\frac{t}{T}\right\rfloor$ and without loss of generality we set $t=0$ at the beginning of an interval during which $\mathcal{G}(t)=\mathcal{G}_{A}$. The Laplacian matrix likewise switches periodically between two constant matrices, i.e., $L(t)=L_{A}\left(L(t)=L_{B}\right)$ when $\mathcal{G}(t)=\mathcal{G}_{A}$ $\left(\mathcal{G}(t)=\mathcal{G}_{B}\right)$. 


\subsection{Model of Consensus Dynamics}

Let $x_{i}$ be a scalar decision variable for agent $i$ in a group of $N$ agents. The continuoustime model of consensus dynamics in which each agent updates its decision variable as a function of the average value of its neighbors is given by

$$
\frac{\mathrm{d}}{\mathrm{d} t} x_{i}= \begin{cases}\frac{1}{\left|\mathcal{N}_{i}(t)\right|} \sum_{j \in \mathcal{N}_{i}(t)}\left(x_{j}(t)-x_{i}(t)\right), & \left|\mathcal{N}_{i}(t)\right| \neq 0 \\ 0, & \left|\mathcal{N}_{i}(t)\right|=0\end{cases}
$$

with initial condition $x_{i}(0)=x_{0_{i}}, i=1, \ldots, N$.

For a time-varying graph $\mathcal{G}(t)$, the dynamics (4) with initial condition $x_{i}(0)=x_{0_{i}}$ converge to a consensus value $x_{i}=x^{*}, i=1, \ldots, N$, for some $x^{*}$ if $\mathcal{G}(t)$ is uniformly connected (Moreau 2005). As described in "Appendix 4," a periodically time-varying graph $\mathcal{G}(t)=\mathcal{G}(t+T)$ is uniformly connected if the union of graphs over a single period of length $T$ is connected.

Following Cao et al. (2008), Swain et al. (2008), we define the rate of convergence to consensus, $\sigma$, as the supremum over all $\bar{\sigma}>0$ for which there exists a $\beta>0$ such that

$$
\left[\sum_{i=1}^{N}\left(x_{i}(t)-x^{*}\right)^{2}\right]^{1 / 2} \leq \beta e^{-\bar{\sigma} t}
$$

for any set of initial conditions. That is, $\sigma$ provides an exponential bound on the rate at which the states approach consensus. If $\mathcal{G}(t)$ is not uniformly connected, then consensus is not achieved and we say that the convergence rate is zero.

It is shown in Swain et al. (2008) that the convergence rate for the periodically switched graph defined in (3) can be expressed analytically as

$$
\sigma=-\frac{1}{T} \log m_{2}
$$

where $m_{2}$ is the second largest eigenvalue of the matrix

$$
M=e^{-(1-p) T L_{A}} e^{-p T L_{B}} .
$$

We can therefore examine the effect of switching between graphs $\mathcal{G}_{A}$ and $\mathcal{G}_{B}$ with duty cycle $p$ by computing the convergence rate as a function of $p$. Note that $p=0$ corresponds to using only $\mathcal{G}_{A}, p=1$ corresponds to using only $\mathcal{G}_{B}$, and $p=\frac{1}{2}$ corresponds to switching between the two with equal interval lengths. If $L_{A}$ and $L_{B}$ are such that the eigenvalues of $e^{L_{A}} e^{L_{B}}$ are the same as the eigenvalues of $e^{L_{A}+L_{B}}$, then we can set $T=1$ with no loss of generality when comparing the relative size of $\sigma$ for switching versus no switching.

\subsection{Results on Switching Configurations and Rate of Convergence to Consensus}

The results of Sect. 3.2 show that coordinated speed oscillations in the three-fish groups generate occlusions that modulate the line-of-sight visibility between fish. We 
(a)

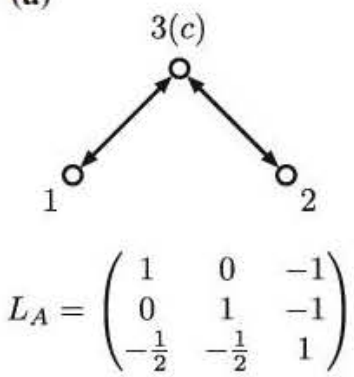

(b)

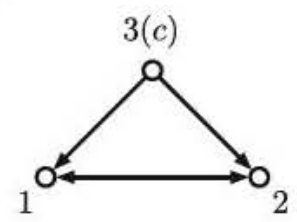

$L_{B}=\left(\begin{array}{ccc}1 & -1 & 0 \\ -1 & 1 & 0 \\ -\frac{1}{2} & -\frac{1}{2} & 1\end{array}\right)$

Fig. 7 Switched sensing topologies A (left) and B (right). The graphs (top) are labeled so that node 3 corresponds to the central fish, and nodes 1 and 2 correspond to leftmost and rightmost neighbors, respectively, i.e., nodes 1 and 2 correspond to the fish from which $\beta_{1}$ and $\beta_{2}$ in Fig. 6 are calculated, respectively. The Laplacian is given for each graph (bottom)

estimate the switching sensing topology that results from the changing line-of-sight visibility and then evaluate the effect of the switching topology on rate of convergence to consensus.

Given the conservative assumptions on which fish gets visual information from which other fish, as described in Sect. 4.1, the V formation and the diagonal formation both produce switching between the same two topologies. This can be seen by observing the arrows on the configurations in Fig. 6, where an arrow is drawn from the fish doing the sensing to the fish being sensed.

Topology A, shown on the left in Fig. 7, corresponds to bidirectional sensing between the center fish and each of the two outer fish and occlusion between the two outer fish. This topology describes the left and nominal configurations in the $\mathrm{V}$ formation and the right and nominal configurations in the diagonal formation as illustrated in Fig. 6.

Topology B, shown on the right in Fig. 7, corresponds to unidirectional sensing from the center fish to each of the two outer fish and bidirectional sensing between the two outer fish (occlusion broken). This topology describes the right configuration in the $\mathrm{V}$ formation and the left configuration in the diagonal formation as illustrated in Fig. 6 ,

The corresponding graph Laplacian matrices for topologies $\mathrm{A}$ and $\mathrm{B}$, denoted $L_{A}$ and $L_{B}$, respectively, are given in Fig. 7. The eigenvalues of $e^{L_{A}} e^{L_{B}}$ are the same as the eigenvalues of $e^{L_{A}+L_{B}}$. So for the convergence rate calculations, we let $T=1$.

We calculate the convergence rate, $\sigma$ as defined by (5), for the A and B topologies and for three values of the duty cycle, $p: p=0, p=1 / 2$, and $p=1$. The duty cycle $p=0$ corresponds to fixed sensing topology $\mathrm{A}$, and duty cycle $p=1$ corresponds to fixed sensing topology B. Duty cycle $p=1 / 2$ corresponds to switching between the two topologies with equal time spent at each. The value of $\sigma$ is calculated using (6), (7), and the graph Laplacian matrices $L_{A}$ and $L_{B}$.

For $p=0$ and $p=1$, the convergence rate is $\sigma=1.0 \mathrm{~s}^{-1}$. For $p=1 / 2$, the convergence rate is $\sigma=1.5 \mathrm{~s}^{-1}$. Therefore, the convergence rate is $50 \%$ faster when the topologies are switching than it is when either topology is used exclusively. It can also be shown that the convergence rate is greater than 1 for any duty cycle value $p \in(0,1)$. Hence, there is a benefit to switching between topologies $A$ and 

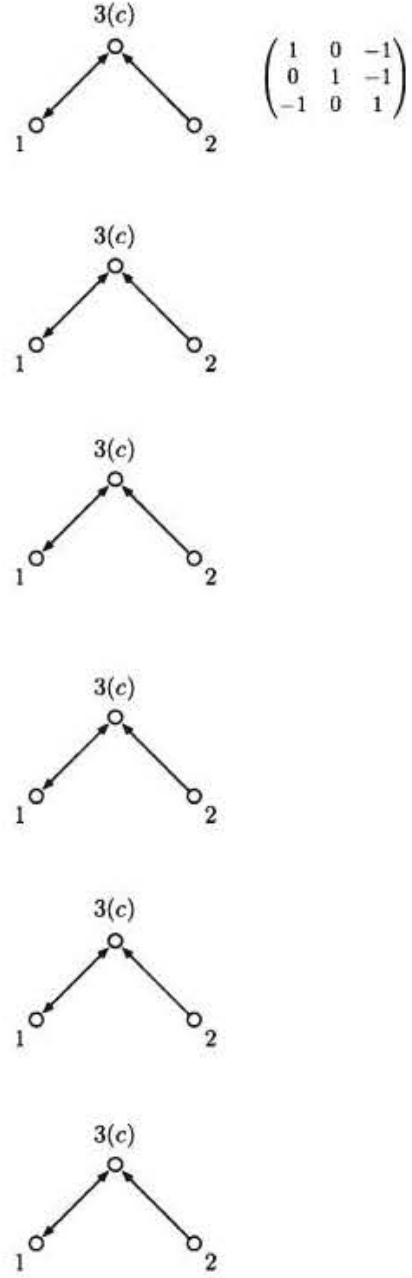

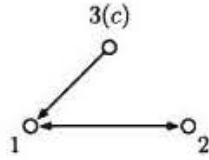

$\left(\begin{array}{ccc}1 & -1 & 0 \\ -1 & 1 & 0 \\ -1 & 0 & 1\end{array}\right)$
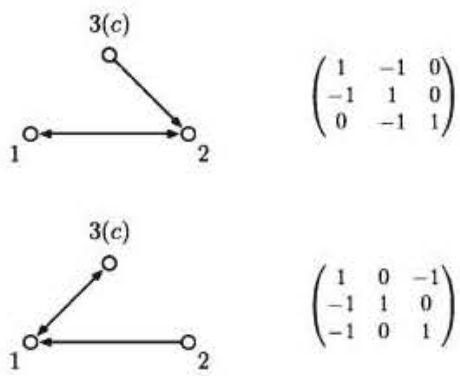

$$
\left(\begin{array}{ccc}
1 & 0 & -1 \\
-1 & 1 & 0 \\
-1 & 0 & 1
\end{array}\right)
$$

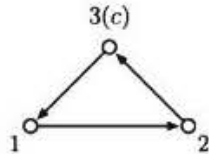

$$
\left(\begin{array}{ccc}
1 & -1 & 0 \\
0 & 1 & -1 \\
-1 & 0 & 1
\end{array}\right)
$$

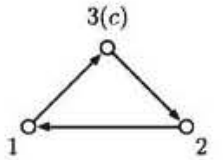

$$
\left(\begin{array}{ccc}
1 & 0 & -1 \\
-1 & 1 & 0 \\
0 & -1 & 1
\end{array}\right)
$$

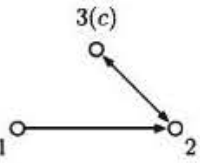

Fig. 8 Switched sensing topologies A (left) and B (right) in which each fish senses only one other fish. Six pairs of graphs are shown from top to bottom. The Laplacian is given for each graph

B over fixing the topology at either A or B no matter what the value of the duty cycle. The convergence rate is maximized for switching when $p=1 / 2$; this maximal convergence rate for switching is equivalent to the convergence rate in the case of an all-to-all and fixed sensing topology, i.e., each fish sensing each other fish at all times.

To test the robustness of this result to the assumptions on sensing among the fish, we compute the convergence rate for six alternative switched sensing topology pairs A and B shown in Fig. 8. These six sensing topology pairs differ from the pair in Fig. 7 in that each fish senses only one other fish at each time instant.

For all the pairs shown in Fig. 8, the convergence rate is $\sigma=1.0 \mathrm{~s}^{-1}$ when $p=0$. Similarly, $\sigma=1.0 \mathrm{~s}^{-1}$ when $p=1$, except for the fourth and fifth pairs (from the top of the figure) in which case $\sigma=1.5 \mathrm{~s}^{-1}$. For switching with duty cycle $p=1 / 2$, 
the convergence rate is $\sigma=1.5 \mathrm{~s}^{-1}$ in every case except for the third pair in which case $\sigma=1.0 \mathrm{~s}^{-1}$. Thus, switching yields $50 \%$ faster convergence than one or both static topologies in all cases except for the third case where switching yields the same convergence as the static cases.

\section{Modeling Collective Dynamics with Coordinated Speed Oscillations}

In this section, we propose an idealized dynamic model for further examination of the remarkable coordinated speed oscillations and collective motion patterns observed in the killifish data and the possibilities for enriched social communication and collective behavior. We show how the analytical tractability of the model enables a systematic investigation of a broad range of conditions and parameterizations of behaviors. Systematic model-based investigation can be used to develop new testable hypotheses for the fish. The model and analysis also make possible a principled approach to biologically inspired design of distributed feedback strategies for coordination of mobile robotic networks that benefit from the enriched social communication studied in this paper.

In the killifish data, the fish are observed to move along approximately straight lines connected by curves near the corners of the tank. The data show that as the fish move together around the curves there are changes among which fish are in front and which are in back. This kind of changing spatial ordering of the fish may provide social communication advantages for the group along the lines of the making and breaking of occlusions discussed in Sects. 3 and 4. For example, if we assume that it is easier for a fish to sense another who is in front rather than in back, then a periodically time-varying change of spatial ordering means an efficient switching among sensing topologies that over time give a well-connected network. The model we develop in this section includes straight-line motion and circular motion of a group with speed oscillations. We show through analysis how the speed oscillations of a group in circular motion yield systematic cycling in spatial ordering. The resulting collective motion patterns also suggest possibilities for enriched spatial density of measurements of an environmental field that can benefit accuracy in collective estimation (Torney et al. 2009; Zhang and Leonard 2010).

\subsection{Continuous-Time Dynamic Model}

Our modeling approach follows that of Sepulchre et al. $(2007,2008)$ with agent-based dynamics, originally with constant speed, augmented here to allow for periodic speed variation and coordination of speed phases. At steady state, each agent in the multiagent system, represented as a particle, has constant (and possibly zero) turning rate and time-periodic speed profile. The model features agent-based steering and speed dynamics that stabilize a large family of collective motion patterns. These patterns realize straight-line motion as well as circular motion of the agents about a common point with coordination among their headings and speed phases. A steered particle model has been used in a variety of analytical work in biology and engineering (including Vicsek et al. 1995; Couzin et al. 2002; Justh and Krishnaprasad 2004). 
We consider a group of $N$ agents and model each agent as a particle of unit mass moving in the plane $\mathbb{R}^{2}$. Extending the notation from Sect. 2, we denote for each agent $k=1, \ldots, N$ at time $t$, the position as $\mathbf{r}_{k}(t)=\left(x_{k}(t), y_{k}(t)\right)$, the velocity as $\mathbf{v}_{k}(t)$, the speed as $s_{k}(t)=\left\|\mathbf{v}_{k}(t)\right\|$, and the heading as $\theta_{k}(t)=L\left\{\mathbf{v}_{k}(t)\right\}$. Then, $\mathbf{v}_{k}=\left(s_{k} \cos \theta_{k}, s_{k} \sin \theta_{k}\right)$. The acceleration of agent $k$ is $\dot{\mathbf{v}}_{k}=\left(\dot{s}_{k} \cos \theta_{k}, \dot{s}_{k} \sin \theta_{k}\right)+$ $\left(-s_{k} \dot{\theta}_{k} \sin \theta_{k}, s_{k} \dot{\theta}_{k} \cos \theta_{k}\right.$ ), where the first term corresponds to the component of force in the direction of motion of the agent and the second term corresponds to the component of force in the direction normal to the agent's velocity.

For ease of notation we identify the real plane $\mathbb{R}^{2}$ with the complex plane $\mathbb{C}$, i.e., the $x$-axis ( $y$-axis) on the real plane corresponds to the real (imaginary) axis on the complex plane. We use bold when we refer to the vector in $\mathbb{R}^{2}$ and no bold when we refer to it as an element in $\mathbb{C}$, e.g., $\mathbf{r}_{k}=\left(x_{k}(t), y_{k}(t)\right) \in \mathbb{R}^{2}$ and $r_{k}=x_{k}+i y_{k} \in \mathbb{C}$, where $i=\sqrt{-1}$. Further, we use the notation $e^{i \theta_{k}}=\cos \theta_{k}+i \sin \theta_{k}$. Then, $v_{k}=\dot{r}_{k}=s_{k} e^{i \theta_{k}}$ and $\dot{v}_{k}=\ddot{r}_{k}=\dot{s}_{k} e^{i \theta_{k}}+s_{k} \dot{\theta}_{k} i e^{i \theta_{k}}$.

Let each agent have a constant unit nominal speed such that $s_{k}(t)=1+\delta s_{k}(t)$. We further let the speed variation $\delta s_{k}$ for each agent $k$ be a $2 \pi$-periodic and zero-mean function of a time-varying speed phase angle $\phi_{k}$, such that $\delta s_{k}\left(\phi_{k}\right)=\delta s_{k}\left(\phi_{k}+2 \pi\right)$.

The steering dynamics of agent $k$ are given by $\dot{\theta}_{k}=\omega+u_{k}(t)$, where $\omega$ is a constant turning rate and $u_{k}$ is a steering control that is zero at steady state. The speed phase dynamics are given by $\dot{\phi}_{k}=\Omega+g_{k}$, where $\Omega$ is a constant describing the intrinsic rate of change of the speed phase and $g_{k}$ is a speed phase control that is zero at steady state. We refer to $\omega$ and $\Omega$ as the natural frequencies of heading and speed phase, respectively.

For conciseness, we specialize to the case of agents with sinusoidal speed oscillations and common amplitude $\mu \in(0,1)$ such that $\delta s_{k}=\mu \cos \phi_{k}$. For details on the development for general periodic speed oscillations with heterogeneous amplitudes see Swain and Leonard (2009), Swain (2012). The steered particle model with controlled speed oscillation phases for agent $k$ is then

$$
\begin{aligned}
\dot{r}_{k} & =\left(1+\mu \cos \phi_{k}(t)\right) e^{i \theta_{k}(t)} \\
\dot{\theta}_{k} & =\omega+u_{k} \\
\dot{\phi}_{k} & =\Omega+g_{k} .
\end{aligned}
$$

We use boldface to represent the ordered vector of the corresponding subscripted quantity, for example, $\mathbf{r}=\left(r_{1}, \ldots, r_{N}\right)^{T} \in \mathbb{C}^{N}$, and $\boldsymbol{\theta}=\left(\theta_{1}, \ldots, \theta_{N}\right) \in T^{N}$. For complex vectors $\mathbf{z}_{1}, \mathbf{z}_{2} \in \mathbb{C}^{N}$, we denote $\overline{\mathbf{z}}_{1}$ to be the complex conjugate of $\mathbf{z}_{1}$ and use the inner product $\left\langle\mathbf{z}_{1}, \mathbf{z}_{2}\right\rangle=\operatorname{Re}\left\{\mathbf{z}_{1}^{T} \overline{\mathbf{z}}_{2}\right\}$.

\subsection{Steady-State Trajectories}

\subsubsection{Parallel Motion}

Solutions of (8) under the steady-state conditions $\dot{\theta}_{k}=\omega=0$ and $\dot{\phi}_{k}=\Omega$ correspond to each agent $k$ moving along a straight line in the direction $\theta_{k}$ with sinusoidal speed and phase $\phi_{k}$. Suppose that the agents have coordinated their headings $\theta_{k}$ to a common 


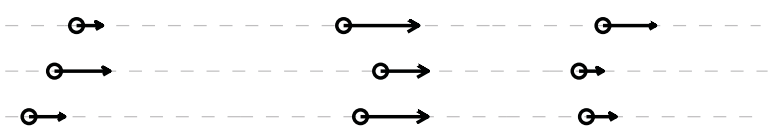

(a)

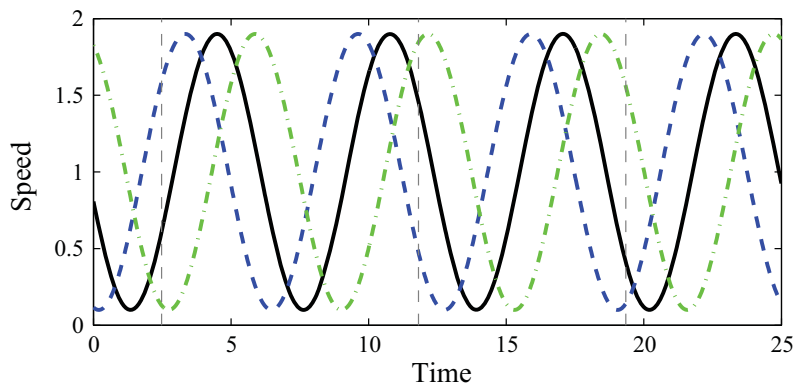

(b)

Fig. 9 Simulation using (8) of steady-state straight-line motion of three agents with speed oscillations with different phases and common heading. a Agent locations (circles) and velocities (arrows) at three time instants corresponding to the three vertical dashed lines in $\mathbf{b}$ the speed of each particle as a function time. From Swain et al. (2007)

direction $\theta$ (e.g., using the feedback $u_{k}$ defined by (12) below with $\kappa_{c}=0, \kappa_{\theta}>0$, and $U$ given by (14)). Then, the agents will move along parallel straight lines that resemble the straight-line motion observed in the killifish data. A simulation example of such a steady-state solution is shown in Fig. 9.

In Swain et al. (2008) we studied these parallel steady-state motions in the case that each agent has range-limited sensing of neighbors and the changing relative positions of agents yield a periodically time-varying sensing network. We defined the notion of an "effective sensing region" as the region that an agent senses over a period of the speed oscillation. In a structured spatial arrangement we showed how to use effective sensing regions to find relative speed oscillation phases and frequency $\Omega$ that maximize the convergence rate of consensus; the analysis is similar to that in Sect. 4.3. We also used effective sensing regions applied to a random initial spatial distribution of agents and showed how periodic relative motion improves network connectivity. Our results in Swain et al. (2008) demonstrate that for a random distribution of agents with periodically time-varying relative positions, connectivity may be achieved with a sensing radius lower than that predicted by percolation theory for agents with fixed relative positions (Quintanilla et al. 2000).

\subsubsection{Circular Motion}

We next examine circular steady-state solutions when $\dot{\theta}_{k}=\omega \neq 0$ and $\dot{\phi}_{k}=\Omega$. Solutions of (8) under these steady-state conditions are given by

$$
r_{k}=c_{k}+R\left(\theta_{k}\right)+\mu E\left(\phi_{k}\right) e^{i \theta_{k}},
$$




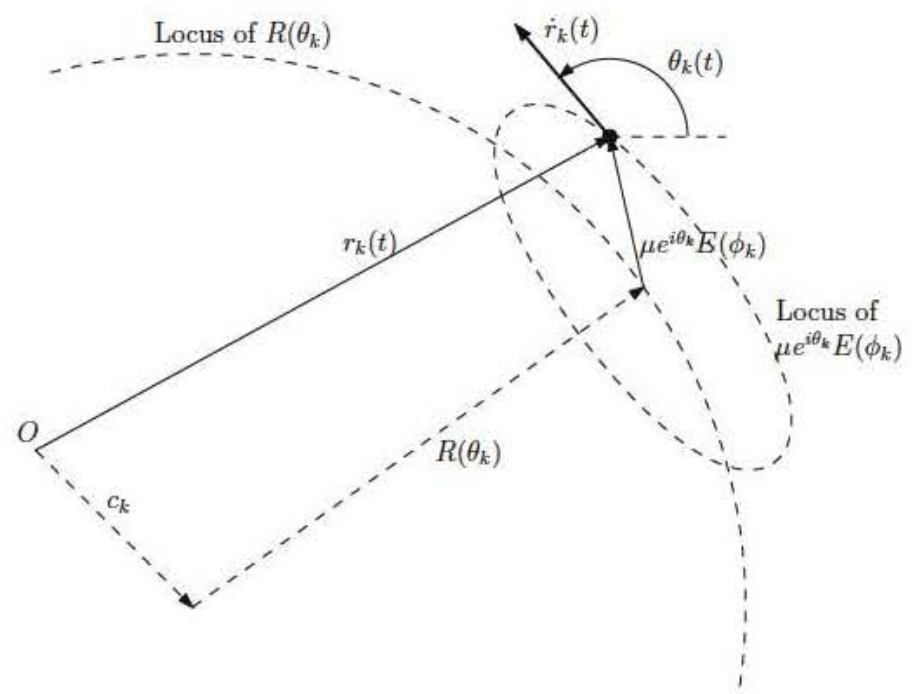

Fig. 10 Decomposition of $r_{k}(t)$ into circular and elliptical components. From Swain et al. (2007)

where $c_{k}$ is a fixed point,

$$
R\left(\theta_{k}\right)=-i \omega^{-1} e^{i \theta_{k}}
$$

defines a circle of radius $\left|\omega^{-1}\right|$, and

$$
E\left(\phi_{k}\right)=\frac{1}{\Omega^{2}-\omega^{2}}\left(\Omega \sin \phi_{k}+i \omega \cos \phi_{k}\right)
$$

defines an ellipse with eccentricity $\frac{\Omega}{\omega}$ and scale $\left|\Omega^{2}-\omega^{2}\right|^{-1}$. The steady-state trajectory of agent $k$, illustrated in Fig. 10, corresponds to motion around an ellipse that rotates around a circle of radius $\left|\omega^{-1}\right|$ centered at $c_{k}$. When $\omega \neq \Omega$ the steady-state orbit remains inside an annulus defined by

$$
\left|\omega^{-1}\right|-\frac{\mu}{\left|\Omega^{2}-\omega^{2}\right|} \leq\left|r_{k}(t)-c_{k}\right| \leq\left|\omega^{-1}\right|+\frac{\mu}{\left|\Omega^{2}-\omega^{2}\right|} .
$$

If $\mu=0$, the orbit is a circle of radius $\left|\omega^{-1}\right|$. If $\mu \neq 0$ then

$$
\frac{\mathrm{d}}{\mathrm{d} t}\left(r_{k}-R_{k}-c_{k}\right)=\frac{\mu}{2}\left(e^{i\left(\theta_{k}+\phi_{k}\right)}+e^{i\left(\theta_{k}-\phi_{k}\right)}\right) .
$$

In the case that $\omega=\Omega$, there is a singularity. The velocity term $\frac{\mu}{2} e^{i\left(\theta_{k}-\phi_{k}\right)}$ in (11) becomes a constant leading to an unbounded spiral trajectory. For $\Omega>\omega$, the ellipse $\mu E\left(\phi_{k}\right)$ has its semimajor axis tangent to the circle $R\left(\theta_{k}\right)$ for each value of $\theta_{k}$ and the orbit has the appearance of a rounded polygon similar to the trajectories of the killifish as they move around the tank (see Fig. 11a). As in Fig. 11a, b, the orbit approximates 
(a)

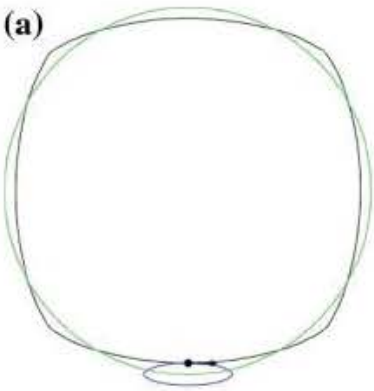

(c)

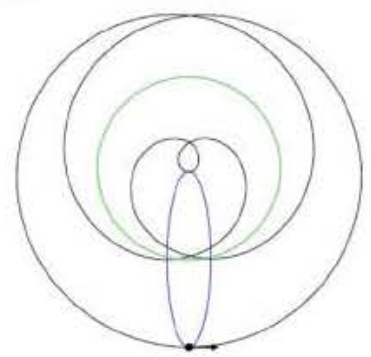

(b)

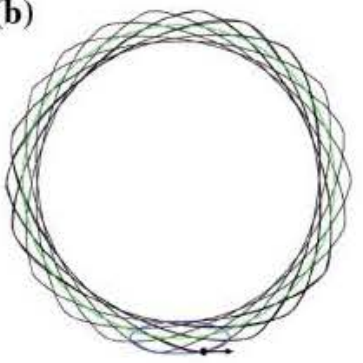

(d)

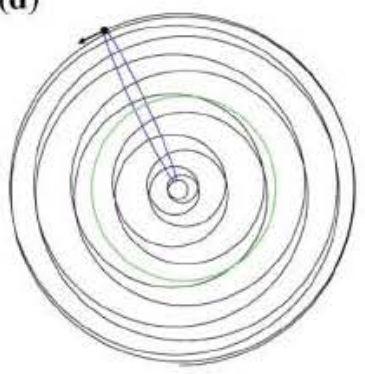

Fig. 11 Simulation using (8) of steady-state motion of a single agent (black curve) for $\omega \neq 0$. The small black filled circle and arrow indicate the agent position and orientation at one time instant. The green curve is the locus of the $R_{k}\left(\theta_{k}\right)$ circle, and the blue curve is the locus of the $\mu E\left(\phi_{k}\right)$ ellipse. The particle moves around the blue ellipse as the center of the blue ellipse moves around the green circle. In all four cases $\mu=0.9$. a A rounded periodic polygon for $\omega=1$ and $\Omega=4$. b An aperiodic trajectory for $\omega=1$ and $\Omega=\pi$.c A periodic cardioid-type trajectory for $\omega=4$ and $\Omega=1$. d An aperiodic cardioid-type trajectory for $\omega=\pi$ and $\Omega=1$

a polygon with $\frac{\Omega}{\omega}$ sides for $\frac{\Omega}{\omega}$ an integer. When $\Omega<\omega$, the ellipse $\mu E\left(\phi_{k}\right)$ has its semimajor axis parallel to the radius vector of the circle $R\left(\theta_{k}\right)$, and the orbit has a cardioid appearance as in Fig. 11c, d. The ratio $\frac{\Omega}{\omega}$ determines the number of loops the orbit completes as $\theta_{k}$ goes through a $2 \pi$ cycle. Whenever $\frac{\Omega}{\omega} \neq 1$ is rational, the orbit is closed and periodic (Fig. 11a, c); otherwise, the orbit is aperiodic (Fig. 11b, d).

\subsection{Stabilization of Coordinated Motion Patterns}

In this section, we complete the model description by defining the steering control $u_{k}$ and speed phase control $g_{k}$; these depend on measurements of neighbors of agent $k$. The collective motion patterns that emerge are the stable steady-state solutions, simply parameterized by the control gains. Let $q_{k}\left(\phi_{k}(t)\right)=\mu E\left(\phi_{k}(t)\right)$. In the case $\omega \neq 0$, we define the instantaneous center of the trajectory of agent $k$ as $c_{k}(t):=$ $r_{k}(t)-R\left(\theta_{k}(t)\right)-q_{k}\left(\phi_{k}(t)\right) e^{i \theta_{k}(t)}$. Then,

$$
\dot{c}_{k}=-u_{k} \omega^{-1}\left(1+i \omega q_{k}\right) e^{i \theta_{k}}-g_{k} \Omega^{-1}\left(v_{k}-i \omega q_{k}\right) e^{i \theta_{k}},
$$

and hence, $c_{k}(t)$ is constant when $u_{k}=g_{k}=0$ as studied in Sect. 5.2. 
Following the approach used in Sepulchre et al. (2007), we use the gradient of a scalar potential $U(\boldsymbol{\theta})$ to define steering control laws that stabilize phase patterns of the headings $\theta_{k}$. Likewise, we use the gradient of a scalar potential $V(\boldsymbol{\phi})$ to define speed phase control laws that stabilize phase patterns of the speed oscillations phases $\phi_{k}$. To include control terms that stabilize the instantaneous centers of each agent to a common point, we make use of the projector $P=I_{N \times N}-\frac{1}{N} \mathbf{1}_{N} \mathbf{1}_{N}^{T}$, where $I_{N \times N}$ is the $N \times N$ identity matrix and $\mathbf{1}_{N}$ is the vector of $N$ ones. Then, $\|P \mathbf{c}\|=0$ is equivalent to $c_{k}=\bar{c} \in \mathbb{C}$, for all $k$.

Theorem 1 (Stability of Coordinated Motion Patterns) Consider $N$ steered agents each with the dynamics (8). Let $U(\boldsymbol{\theta})$ and $V(\boldsymbol{\phi})$ be two twice differentiable phase potentials. Assume that $U: T^{N} \rightarrow\left[0, U_{\max }\right]$ for some scalar $U_{\max }>0$ such that $\left\langle\operatorname{grad} U, \mathbf{1}_{N}\right\rangle=0$. Assume that $V: T^{N} \rightarrow\left[0, V_{\max }\right]$ for some scalar $V_{\text {max }}>0$ such that $\left\langle\operatorname{grad} V, \mathbf{1}_{N}\right\rangle=0$. Define the steering control

$$
u_{k}=\kappa_{c} \omega^{-1}\left\langle P_{k} \mathbf{c},\left(1+i \omega q_{k}\right) e^{i \theta_{k}}\right\rangle-\kappa_{\theta} \frac{\partial U}{\partial \theta_{k}}
$$

and the speed phase control

$$
g_{k}=\kappa_{c} \Omega^{-1}\left\langle P_{k} \mathbf{c},\left(v_{k}-i \omega q_{k}\right) e^{i \theta_{k}}\right\rangle-\kappa_{\phi} \frac{\partial V}{\partial \phi_{k}}
$$

where $\kappa_{c}>0, \kappa_{\theta}$, and $\kappa_{\phi}$ are real constants. These controls asymptotically stabilize the group of $N$ agents to a steady-state pattern of heading phases $\boldsymbol{\theta}$ in the critical set of $U(\boldsymbol{\theta})$ when $\kappa_{\theta} \neq 0$, to a steady-state pattern of speed oscillation phases $\boldsymbol{\phi}$ in the critical set of $V(\boldsymbol{\phi})$ when $\kappa_{\phi} \neq 0$, and to a steady center $c_{k}=\bar{c}=\frac{1}{N} \sum_{k=1}^{N} c_{k}$, for each agent $k$. Furthermore, the steady-state pattern of $\boldsymbol{\theta}$ (respectively $\boldsymbol{\phi}$ ) corresponds to a local maximum of $U$ (respectively $V$ ) when $\kappa_{\theta}>0$ (respectively $\kappa_{\phi}>0$ ) and a local minimum of $U$ (respectively $V$ ) when $\kappa_{\theta}<0$ (respectively $\left.\kappa_{\phi}<0\right)$. For $\kappa_{c}=0$, the steady-state pattern corresponds to straight-line motion.

The proof is similar to the proofs in Sepulchre et al. (2007); the detailed proof for the general periodic speed oscillation case is given in Section 4.3 of Swain (2012). It can be shown that the control laws (12) and (13) depend only on relative measurements. Additionally, following the approach in Sepulchre et al. (2008), the stability result can be extended to the case in which the inter-agent sensing topology is defined by a possibly time-varying graph that reflects limitations on sensing.

\subsection{Examples of Coordinated Motion Patterns}

We illustrate for the case that resembles the killifish in which the heading directions are synchronized and the speed oscillation phases differ. The headings will be synchronized when the speed of the center of mass of the group is maximized. The average speed of the group is proportional to the magnitude of 


$$
p_{\theta}=\frac{1}{N} \sum_{k=1}^{N} e^{i \theta_{k}}
$$

which is equivalent to the complex-order parameter (Kuramoto 1984; Strogatz 2000). Define the heading phase potential as

$$
U(\theta)=\frac{N}{2}\left|p_{\theta}\right|^{2}
$$

By Theorem 1 the speed of the center of mass $\left|p_{\theta}\right|$ is maximized using $u_{k}$ given by (12) with $\kappa_{\theta}>0$.

To stabilize the speed oscillation phases to a pattern in which they are uniformly distributed around the circle, following Sepulchre et al. (2007), we use $\kappa_{\phi}<0$ and minimize the speed oscillation phase potential

$$
V(\phi)=\sum_{m=1}^{\left\lfloor\frac{N}{2}\right\rfloor}\left|\frac{1}{m N} \sum_{j=1}^{N} e^{i m \phi_{j}}\right|^{2}
$$

Figure 12 illustrates the coordinated motion pattern for four agents with the dynamics (8), steering control (12), speed phase control (13) with potentials (14) and (15) and $\kappa_{\theta}>0$ and $\kappa_{\phi}<0$. For each agent we use the parameters $\mu=0.9, \omega=1$, and $\Omega=4$, which are the same as in Fig. 11a where the trajectory most closely resembles the killifish. Figure 12a illustrates the steady solution, and Fig. 12b shows a simulation. At steady state, the four agents move around an ellipse, with uniform spacing, while the center of the ellipse moves around a fixed circle. In the snapshot shown in Fig. 12a, the purple agent is in front, the cyan agent is in the inside of the curve,

(a)

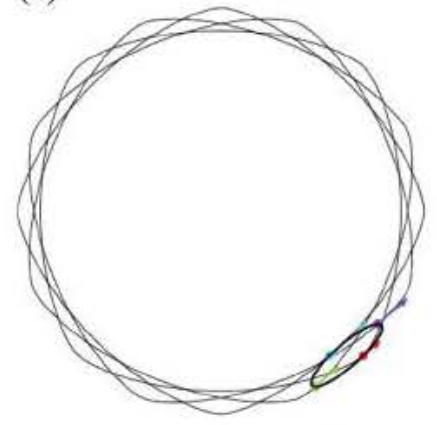

(b)

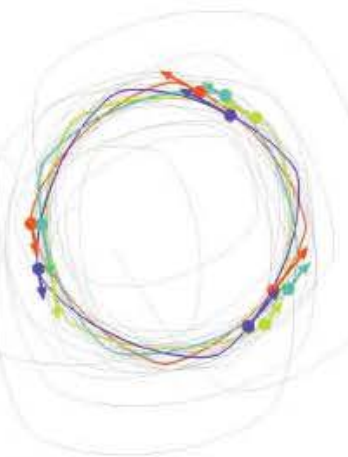

Fig. 12 Coordinated motion pattern for $N=4$ agents with dynamics (8). Steering control is given by (12), and speed oscillation phase control is given by (13) with potentials (14) and (15) and $\kappa_{\theta}>0, \kappa_{\phi}<0$, $\mu=0.9, \omega=1$, and $\Omega=4$. Trajectories are shown in black. In snapshots of the four agents, the positions are shown as colored circles. The colored arrows show heading direction with the length of the arrow inversely proportional to corresponding agent's instantaneous speed. a Steady-state solution. b Simulated trajectories from random initial conditions (from Swain et al. 2007). Three snapshots of the four vehicles are superimposed 
the green agent is in back, and the red agent is on the outside of the curve. It can be seen that all agents head in the same direction. At the instant shown, the cyan agent is slowest since it moves on the inside, while the red agent is fastest since it moves on the outside of the circle. The snapshots in Fig. 12b show how the individuals regularly change position around the ellipse, i.e., each gets time in the front and in the back of the group, and on the inside and the outside of the curve. For direction-limited sensing, this implies a regular switching among sensing topologies with strong connectivity over a period. Further, in the case that each agent samples an external environmental field, the rotation of the group about an ellipse that moves around a circle provides for spatially dense collective measurements and accuracy in collective estimates of spatial gradients in the field. The collective motion pattern also provides redundancy of measurements, e.g., if the group is tracking a circular boundary, then every agent will take measurements on both sides of the boundary.

A comprehensive study of the coordinated motion patterns that can be stabilized using the dynamics described in this section is provided in Swain (2012). It is also shown in Swain (2012) how to systematically solve for the agent control laws given a desired collective motion pattern.

\section{Discussion}

Our results show quantitatively that the killifish in two-fish and three-fish schools commonly exhibit sustained coordinated speed oscillations such that pairs of fish are close to anti-synchronized in phase. The speed oscillations are quantified using a novel method for locating segments of oscillating speed, where speed is calculated from trajectory data acquired by digitally tracking the fish from overhead video and oscillations are classified using the Hilbert transform. That the speed oscillations are coordinated among the fish can be inferred from the correlated periods of oscillation with very similar frequency among the fish and the consistent oscillation phase difference.

The coordinated oscillating speeds lead to oscillations in relative positions among fish; at the group level this corresponds to cycling of the spatial configuration. In both the two-fish and the three-fish schools, the fish are observed to cycle closer and then farther from their neighbors. We hypothesize that the resulting periodic changes in school configurations have a positive impact on passing of socially sensed information; when social sensing depends on relative position or velocity, cyclic relative motion can be expected to increase the richness of sensed measurements.

We consider social communication through visual sensing, and we examine the periodically time-varying configurations in the three-fish schools. We estimate a switching sensing topology that describes which fish can see which other fish in the three-fish schools as occlusions are made and broken periodically in time. We evaluate the switching sensing topology in a novel way by computing the rate of convergence to consensus (for a decision variable such as heading direction) in a dynamic system of decision makers with network structure defined by the switching sensing topology. For the purposes of comparison we compute the rate of convergence to consensus for the same consensus dynamics in the case that the network structure is static. Among static sensing topologies, the maximum rate of convergence to consensus is achieved 
in the all-to-all case, i.e., when each individual senses every other individual. However, an all-to-all topology is often not possible when sensing depends on the spatial distribution of the group, and even if possible might be costly in terms of attention. For lattices and other regular patterns, the visual sensing topology determined by line-of-site visibility will be limited due to occlusions.

We compute the rate of convergence to consensus in the case that the topology switches between the two sensing topologies estimated from the three-fish school data. By this metric, switching outperforms a fixed topology at either of the two estimated sensing topologies; the speed of convergence is as much as $50 \%$ more than what could be achieved with one of the fixed topologies, and maximized when the time is equally split between the two topologies. This maximal convergence rate is equal to the convergence rate in the case of the static all-to-all topology, but achieved with limited sensing topologies. We show further that the results are robust to changes in assumptions on numbers of fish sensed at any given moment. These results suggest the possibility of an evolutionary advantage to coordinated relative motion in this context.

We find another striking benefit of coordinated relative motion with respect to social communication by showing that the periodic relative motion of the killifish facilitates periodic cycling between different relative positions that are individually optimal with respect to different benefits. The relative positions of the killifish correspond to relative bearings from a focal fish to a neighbor fish that have been predicted by Dill et al. (1997) to be optimal with respect to the focal fish's ability to detect changes in the heading or speed of a neighbor. It was suggested that these values may predict the average relative bearings observed in animal groups. We find that the killifish cycle about a range of relative bearings that includes three of the four optimal angles predicted by Dill et al. (1997). The three optimal angles exhibited by the killifish are (1) the bearing angle $35.3^{\circ}$, which maximizes sensitivity to speed and heading changes of the visual cue for detecting time to collision, (2) the bearing angle $45.0^{\circ}$, which maximizes sensitivity to a heading change of the visual cue for detecting angular velocity, and (3) $63.4^{\circ}$, which is the bearing angle that maximizes sensitivity to a speed change of the visual cue for detecting loom.

We have derived an analytically tractable model of a network of agents moving with oscillating speed and demonstrated its use in systematically studying coordinated motion patterns along straight lines and around circles. For agents modeled with rangelimited sensing, periodic relative motion yields enhanced effective sensing, enriching social communication. Further, in circular motion, agents modeled with out-of-phase sinusoidal speed oscillations will rotate periodically in time around an ellipse that rotates around the circle. Each agent repeatedly takes a turn being in the front and back of the group and in the inside and outside of the circle of motion. Thus, communication is enriched not only for range-limited sensing but also for direction-limited sensing since each agent switches between seeing and being seen. For the group measuring a distributed environmental field, the elliptical circulation provides high spatial density of measurements by the group. For example, it can improve their accuracy in estimating the gradient of the measured field or tracking a level set or boundary of a resource patch (Zhang and Leonard 2010). This collective motion also provides redundancy in measurements, particularly useful in the case of a sensor failure. 
While we have primarily considered the effects of relative motion on the ability of visual information to pass between fish, relative motion is also linked to the quality of visual information. For example, optical flow is generated on the retina of the focal fish when that fish moves relative to its neighbors. Periodicity of relative motion, induced by coordinated (anti-synchronized) speed oscillations, implies periodicity of the optical flow, which may improve signal-to-noise ratio with regard to detecting the position and motion of other fish from visual information that also includes noise from the environment. Likewise, there may be benefit in lateral line sensing due to unsteady fluid flow between the fish as a result of the relative motion induced by coordinated speed oscillations.

Our results show quantitatively ways in which periodically changing group configurations can enrich social communication as compared to static configurations. The techniques we describe are directly applicable to other settings in which coordinated periodic relative motion is present. For example, they could prove useful to analyze burst-and-coast swimming in fish schools where it is not clear whether or not bursting and coasting provides an energetic advantage (Weihs 1974; Fish et al. 1991). The authors of Fish et al. (1991) propose that any energetic advantage may be incidental to benefits associated with maintaining position within the school. This may have some connections to the case of the killifish: The speed oscillations require active deceleration which is most likely energetically disadvantageous, but at the same time the speed oscillations provide a benefit in information passing.

Animal groups that move together exhibit remarkable behavior despite limitations in the capabilities of individuals. Our results provide new insights and motivate further study on how the role of relative motion can be used to explain collective behavior in animal groups and to design collective behavior in engineered groups such as mobile robotic networks.

Acknowledgments The authors gratefully acknowledge support from Office of Naval Research Grants N00014-09-1-1074 and N00014-14-1-0635, and Army Research Office Grants W911NG-11-1-0385 and W911NF-14-1-0431. IDC thanks Jens Krause and Jean-Guy Godin for advice on the experiments and access to experimental facilities, Albert Kao for help with preliminary digitization and analysis, and support from the National Science Foundation (NSF) Grants IOS-1355061, and EAGER IOS-1251585, and Human Frontier Science Project Grant RGP0065/2012.

\section{Appendix}

\section{Appendix 1: Experiments and Trajectory Data}

Groups of two and three killifish, collected from Silver Lake, Sackville, New Brunswick, Canada, were among the small schools filmed by IDC in September 2000, while they swam in a $155-\mathrm{cm}$ square tank in shallow water $(2.5 \mathrm{~cm})$. The smallest of the fish were on average $3 \mathrm{~cm}$ long, and the largest were on average $4 \mathrm{~cm}$ long. The video was recorded from $190 \mathrm{~cm}$ above the tank on a Sony DVCAM at 31.2 frames per second and was later transferred to DV-format video files. Custom tracking software (Swain 2011; Swain et al. 2012) was used to extract raw trajectory data from the video files of the groups of two and three killifish. The output of the tracking software is one 
trajectory time series for each fish in each video. That is, for each fish in each video with $N_{t}$ frames we have

$$
\mathbf{r}(t)=\left[\begin{array}{l}
x(t) \\
y(t)
\end{array}\right], t=\left\{0,1, \ldots, N_{t}-1\right\}
$$

where $(x(t), y(t))$ are the coordinates, in meters, of the centroid of the fish relative to the bottom left corner of the video frame at time $t T_{s}$, and $T_{S}=32.1 \mathrm{~ms}$ is the time between video frames.

From these data we calculate positions in meters by multiplying image coordinates in pixels by a scaling factor, that is, the ratio of the known width of the tank in meters to the width of its image in pixels. This scaling conversion is sufficient because there was very little lens distortion and because the camera was mounted such that the image plane was aligned with the plane of the bottom of the tank (i.e., there was negligible rotation between the image coordinate system and the tank coordinate system).

A subset of the trajectory time series data is used for the analysis of speed oscillations (see Sect. 2). To select this subset, a set of candidate data sequences is determined by rejecting time samples for which either a) one or more fish is not visible in the video frame or b) the fastest-swimming fish has a speed of less than $4 \mathrm{~cm} / \mathrm{s}$ (i.e., the fish were idle). Any of these candidate segments that is less than $2.0 \mathrm{~s}$ in duration is rejected. A subset of the remaining sequences is selected in order to balance the number of time sample contributions from each experimental group. First, the group with the smallest total number of candidate time samples is rejected. Sequences were then selected from each of the remaining groups, one group at a time and in the order in which they were recorded, until the number of samples for that group exceeds the number of samples from the second smallest group. This method produces a similar number of samples from each group. It is applied separately to the two-fish data and the three-fish data.

For the analysis of speed oscillations in two-fish schools, the method provides 15 groups of fish (after the group with the fewest available samples was removed) with a total of 130 time segments and a total duration of $1343.40 \mathrm{~s}$ (22.39 $\mathrm{min})$. The time contribution per group ranges from 89.94 to $110.51 \mathrm{~s}$ with shortest segment 2.08 $\mathrm{s}$, longest segment $40.26 \mathrm{~s}$, and average segment $10.33 \mathrm{~s}$. For the analysis of speed oscillations in three-fish schools, the method provides 11 groups of fish (after the group with the fewest available samples is removed) with a total of 63 time segments and a total duration of $912.63 \mathrm{~s}(15.21 \mathrm{~min})$. The time contribution per group ranges from 82.95 to $108.14 \mathrm{~s}$ with shortest segment $2.08 \mathrm{~s}$, longest segment $61.31 \mathrm{~s}$, and average segment $14.49 \mathrm{~s}$.

A subset of the trajectory time series three-fish data is used for analysis of configurations (see Sect. 3). This subset is determined in a manner similar to that described above with the same constraints on visibility and minimum speed. In addition, segments during which any fish is within three body lengths $(12.0 \mathrm{~cm})$ of the edge of the tank or with duration less than $2.0 \mathrm{~s}$ are rejected. Of the remaining segments, only those during which at least two of the fish engage in coordinated speed oscillations are kept. These segments are determined according to the algorithm described in Sect. 2.1 for isolating oscillatory segments in a time series using the instantaneous phase of 
the relative speed between a pair of fish. The number of time samples from each experimental group is balanced using the procedure described above.

For the analysis of configurations in three-fish schools, the method provided 11 groups of fish (after the group with the fewest available samples was removed) with a total of 246 segments and a total duration of $1086.76 \mathrm{~s}$ (18.11 min). The time contributed per group ranges from 105.83 to $115.03 \mathrm{~s}$ with shortest segment $1.12 \mathrm{~s}$, longest segment $15.45 \mathrm{~s}$, and average segment $4.42 \mathrm{~s}$.

\section{Appendix 2: Velocity Estimation}

We describe the method we use to estimate the velocity, $\mathbf{v}(t)$, and the nominal speed, $\bar{s}(t)$, of each fish at time $t$ during each segment of the trajectory data for the two-fish and three-fish groups.

The velocity, $\mathbf{v}(t)$, is estimated by filtering the "raw" velocity $\overline{\mathbf{v}}(t)$ computed from the forward differences in position as

$$
\overline{\mathbf{v}}(t)=\frac{1}{T_{S}}(\mathbf{r}(t+1)-\mathbf{r}(t))
$$

Filtering is achieved by convolving $\overline{\mathbf{v}}(t)$ with a truncated sinc kernel that approximates an ideal low-pass filter in the frequency domain [(see for example Proakis and Manolakis (1996)]. The filter kernel, $K(\tau)$, is the sequence

$$
K(\tau)=\bar{K} \frac{\sin 2 \pi \tau T_{s} f_{c}}{2 \pi \tau T_{s} f_{c}}, \quad-\frac{L_{K}-1}{2} \leq \tau \leq \frac{L_{K}-1}{2}
$$

where $f_{c}=2.0 \mathrm{~Hz}$ is the cutoff frequency, $L_{K}=201$ is an odd integer equal to the number of samples in the kernel, and $\bar{K}$ is a constant that satisfies $\sum_{\tau=-\frac{L_{K}-1}{2}}^{\frac{L_{K}-1}{2}} K(\tau)=$ 1. The cutoff frequency $f_{c}$ is chosen to eliminate as much noise as possible from the sample speed sequences without over-smoothing the estimate.

The convolution operation is undefined for the first and last $\left(L_{K}-1\right) / 2$ samples of the raw velocity sequence. To avoid losing these samples, the raw velocity sequence is extended beyond its original length, $N_{t}$. The extended raw velocity is defined as

$$
\overline{\mathbf{v}}_{e}(t)= \begin{cases}\overline{\mathbf{v}}(-t), & -\frac{L_{K}-1}{2} \leq t<0 \\ \overline{\mathbf{v}}(t), & 0 \leq t<N_{t} \\ \overline{\mathbf{v}}\left(2 N_{t}-t\right), & N_{t} \leq t<N_{t}+\frac{L_{K}-1}{2}\end{cases}
$$

The convolution of the extended raw velocity sequence with the kernel sequence is taken as the estimate of the velocity:

$$
\mathbf{v}(t)=\sum_{\tau=-\frac{L_{K}-1}{2}}^{\frac{L_{K}-1}{2}} \overline{\mathbf{v}}_{e}(\tau-t) K(\tau), 0 \leq t<N_{t} .
$$


The speed is $s(t)=\|\mathbf{v}(t)\|$ and the heading is $\theta(t)=L\{\mathbf{v}(t)\}$. The nominal speed, $\bar{s}(t)$, is calculated by applying a low-pass filter with bandwidth $0.375 \mathrm{~Hz}$ to the raw velocity and taking the Euclidean norm of the resulting vector. A sinc kernel of the form (16) is used with $f_{c}=0.375 \mathrm{~Hz}$ and $L_{K}=1067$ samples. Sequences are extended as in (17) prior to filtering.

\section{Appendix 3: Metric for Speed Oscillation Phase Difference}

The mean sign product (MSP) is derived to provide a measure of coordination of speed oscillations between a pair of fish that is not so sensitive to amplitude fluctuations and noise in the speed variation signals. The MSP is computed as a function of the relative speed variation $\delta s_{i j}$ for the pair of fish $i$ and $j$, where $\delta s_{i j}(t)=\delta s_{i}(t)-\delta s_{j}(t)$. We define

$$
\operatorname{MSP}(i, j)=\frac{1}{N_{t}} \sum_{t=1}^{N_{t}} \operatorname{sign}\left\{\left(\delta s_{i}(t)-\delta \bar{s}_{i}\right)\left(\delta s_{j}(t)-\delta \bar{s}_{j}\right)\right\},
$$

where $\delta \bar{s}_{i}\left(\delta \bar{s}_{j}\right)$ is the average value of $\delta s_{i}(t)\left(\delta s_{j}(t)\right)$ over the segment.

When fish $i$ and fish $j$ have speed oscillations that differ in phase by $180^{\circ}$, then $\operatorname{MSP}(i, j)=-1$, and we say that their speeds are anti-synchronized. When fish $i$ and fish $j$ have speed oscillations that differ in phase by $0^{\circ}$, then $\operatorname{MSP}(i, j)=1$, and we say that their speeds are synchronized.

For perfect sinusoids, the MSP varies linearly with the phase difference, $\Delta \phi$, modulo $360^{\circ}$, as MSP $=1-2\left|\frac{\Delta \phi}{180^{\circ}}\right|$. Noise tends to compress the estimate away from the \pm 1 extremes. $\operatorname{MSP}(i, j)<0$ indicates that the speed oscillations of fish $i$ and fish $j$ are more anti-synchronized than they are synchronized. Likewise, $\operatorname{MSP}(i, j)>0$ indicates that the speed oscillations of fish $i$ and fish $j$ are more synchronized than they are anti-synchronized. $\operatorname{MSP}(i, j)=0$ corresponds to a phase difference of $\pm 90^{\circ}$. The variance of the MSP, calculated across subsegments of equal length, is an indication of the uniformity of phase differences. If there is no uniformity, then the mean MSP tends toward zero and the variance tends toward 1/3 (i.e., the variance of a uniform distribution over the range -1 to 1 ). If there is a high degree of uniformity in phase difference, then the variance will be low.

\section{Appendix 4: Uniform Connectedness of a Periodically Time-Varying Graph}

Consider a time-varying graph $\mathcal{G}(t)$ defined by a node set $\mathcal{V}=\{1, \ldots, N\}$ and an edge set $\mathcal{E}(t) \subseteq \mathcal{V} \times \mathcal{V}$. A node $i$ is said to be connected to node $j$ at time $t$ if there is a path in $\mathcal{G}(t)$ from $i$ to $j$ that respects edge directions, i.e., there is a set of nodes $k_{1}, \ldots, k_{n} \in \mathcal{V}, n \leq N-2$, such that $\left(i, k_{1}\right),\left(k_{1}, k_{2}\right), \ldots,\left(k_{n}, j\right)$ are all in $\mathcal{E}(t)$. The graph $\mathcal{G}(t)$ is defined to be connected at time $t$ if there is a node $k \in \mathcal{V}$ that is connected to all other nodes at time $t$. For a time-varying graph $\mathcal{G}(t)$ and any time interval $I$, we may construct a graph $\mathcal{G}_{I}$, where $\mathcal{G}_{I}$ has the same node set, $\mathcal{V}$, as $\mathcal{G}(t)$ and an edge set $\mathcal{E}_{I}$ defined such that $(i, j) \in \mathcal{E}_{I}$ if and only if $(i, j) \in \mathcal{E}(t)$ for some $t \in I$. We say that node $i$ is connected over time interval $I$ to node $j$ if there is a path 
from $i$ to $j$ in $\mathcal{G}_{I}$ that respects edge directions. A graph $\mathcal{G}(t)$ is said to be uniformly connected if there is a node $k \in \mathcal{V}$ and a time horizon $\tau>0$ such that, for all $t \geq 0$, node $k$ is connected to all other nodes over the interval $I=[t, t+\tau]$. If the graph is periodically time varying with period $T$, i.e., $\mathcal{G}(t)=\mathcal{G}(t+T)$, then $\mathcal{G}(t)$ is uniformly connected if the graph $\mathcal{G}_{I}$ is connected for $I=[t, t+T]$ for any $t \geq 0$.

\section{References}

Bajec, I., Heppner, F.: Organized flight in birds. Anim. Behav. 78(4), 777-789 (2009)

Boashash, B.: Estimating and interpreting the instantaneous frequency of a signal. I. Fundamentals. Proc. IEEE 80(4), 520-538 (1992)

Breder Jr, C.M.: Fish schools as operational structures. Fish. Bull. 74(3), 471-502 (1976)

Cao, M., Morse, A.S., Anderson, B.D.O.: Reaching a consensus in a dynamically changing environment: convergence rates, measurement delays, and asynchronous events. SIAM J. Control Optim. 47(2), 601-623 (2008)

Chagnaud, B.P., Coombs, S.: Information encoding and processing by the peripheral lateral line system. In: Coombs, S., Bleckmann, H., Fay, R.R., Popper, A.N. (eds.) The Lateral Line System. Springer, New York (2014)

Couzin, I.D., Krause, J., James, R., Ruxton, G., Franks, N.: Collective memory and spatial sorting in animal groups. J. Theor. Biol. 218, 1-11 (2002)

Couzin, I.D., Krause, J., Franks, N.R., Levin, S.A.: Effective leadership and decision-making in animal groups on the move. Nature 433, 513-516 (2005)

Czirok, A., Barabasi, A.L., Vicsek, T.: Collective motion of self-propelled particles: kinetic phase transition in one dimension. Phys. Rev. Lett. 82(1), 209-212 (1999)

Dill, L.M., Holling, C.S., Palmer, L.H.: Predicting the three-dimensional structure of animal aggregations from functional considerations: the role of information. In: Parrish, J.K., Hamner, W.M. (eds.) Animal Groups in Three Dimensions, pp. 207-224. Cambridge University Press, Cambridge (1997)

Fish, F.E., Fegeley, J.F., Xanthopoulos, C.J.: Burst-and-coast swimming in schooling fish (Notemigonus Crysoleucas) with implications for energy economy. Comp. Biochem. Physiol. 100A(3), 633-637 (1991)

Gueron, S., Levin, S.A., Rubenstein, D.I.: The dynamics of herds: from individuals to aggregations. J. Theor. Biol. 182(1), 85-98 (1996)

Hanke, W., Lauder, G.V.: Fish schooling: 3D kinematics and hydrodynamics. Integr. Comp. Biol. 46(Supp 1), E54 (2006)

Jadbabaie, A., Lin, J., Morse, A.S.: Coordination of groups of mobile autonomous agents using nearest neighbor rules. IEEE Trans. Autom. Control 48(6), 988-1001 (2003)

Justh, E.W., Krishnaprasad, P.S.: Equilibria and steering laws for planar formations. Syst. Control Lett. 52(1), 25-38 (2004)

Krause, J., Hoare, D.J., Croft, D., Lawrence, J., Ruxton, G.D., Ward, A., Godin, J.G., James, R.: Fish shoal composition: mechanisms and constraints. Proc. R. Soc. B Biol. Sci. 267(1456), 2011-2017 (2000)

Kuramoto, Y.: Chemical Oscillations, Waves, and Turbulence. Springer, Berlin (1984)

Leonard, N.E., Shen, T., Nabet, B., Scardovi, L., Couzin, I.D., Levin, S.A.: Decision versus compromise for animal groups in motion. Proc. Natl. Acad. Sci. 109(1), 227-232 (2012)

Moreau, L.: Stability of multiagent systems with time-dependent communication links. IEEE Trans. Autom. Control 50(2), 169-182 (2005)

Olfati-Saber, R., Murray, R.M.: Consensus protocols for networks of dynamic agents. In: IEEE, Proceedings of the American Control Conference, pp. 951-956. (2003)

Parrish, J., Turchin, P.: Individual decisions, traffic rules, and emergent pattern in schooling fish. In: Parrish, J.K., Hamner, W.M. (eds.) Animal Groups in Three Dimensions, pp. 126-141. Cambridge University Press, Cambridge (1997)

Partridge, B.L., Pitcher, T., Cullen, J.M., Wilson, J.: The three-dimensional structure of fish schools. Behav. Ecol. Sociobiol. 6(4), 277-288 (1980)

Proakis, J.G., Manolakis, D.G.: Digital Signal Processing, 3rd edn. Prentice Hall, Englewood Cliffs (1996)

Quintanilla, J., Torquato, S., Ziff, R.M.: Efficient measurement of the percolation threshold for fully penetrable discs. J. Phys. A Math. Gen. 33, L399-L407 (2000) 
Sepulchre, R., Paley, D.A., Leonard, N.E.: Stabilization of planar collective motion: all-to-all communication. IEEE Trans. Autom. Control 52(5), 811-824 (2007)

Sepulchre, R., Paley, D.A., Leonard, N.E.: Stabilization of planar collective motion with limited communication. IEEE Trans. Autom. Control 53(3), 706-719 (2008)

Spooner, G.M.: Some observations in schooling in fish. J. Mar. Biol. Assoc. U.K. 17(2), 421-448 (1931)

Srivastava, V., Leonard, N.E.: Collective decision-making in ideal networks: the speed-accuracy tradeoff. IEEE Trans. Control Netw. Syst. 1(1), 121-130 (2014)

Strandburg-Peshkin, A., Twomey, C.R., Bode, N.W., Kao, A.B., Katz, Y., Ioannou, C.C., Rosenthal, S.B., Torney, C.J., Wu, H., Levin, S.A., Couzin, I.D.: Visual sensory networks and effective information transfer in animal groups. Curr. Biol. 23(17), R709-711 (2013)

Strogatz, S.H.: From Kuramoto to Crawford: exploring the onset of synchronization in populations of coupled oscillators. Phys. D 143, 1-20 (2000)

Sumpter, D., Buhl, J., Biro, D., Couzin, I.: Information transfer in moving animal groups. Theory Biosci. 127(2), 177-186 (2008)

Swain, D.T., Cao, M., Leonard, N.E.: Effective sensing regions and connectivity of agents undergoing periodic relative motions. In: IEEE, Proceedings of the 47th IEEE Conference on Decision and Control. pp. 3089-3094. (2008)

Swain, D.T., Leonard, N.E., Couzin, I.D., Kao, A., Sepulchre, R.J.: Alternating spatial patterns for coordinated group motion. In: IEEE, Proceedings of the 46th IEEE Conference on Decision and Control. pp. 2925-2930. (2007)

Swain, D.T., Leonard, N.E.: On the trajectories and coordination of steered particles with time-periodic speed profiles. In: IEEE, Proceedings of the American Control Conference, pp. 1286-1291. (2009)

Swain, D.T.: MADTraC framework source documentation. http://poincare.princeton.edu/mt/docs/ (2011)

Swain, D.T.: The Role of the Dynamics of Relative Motion in Information Passing in Natural and Engineered Collective Motion. In: $\mathrm{PhD}$ thesis, Dept. of Mechanical and Aerospace Engineering, Princeton University (2012)

Swain, D.T., Couzin, I.D., Leonard, N.E.: Real-time feedback-controlled robotic fish for behavioral experiments with fish schools. Proc. IEEE 100(1), 150-163 (2012)

Torney, C., Neufeld, Z., Couzin, I.D.: Context-dependent interaction leads to emergent search behavior in social aggregates. Proc. Natl. Acad. Sci. 106(52), 22055-22060 (2009)

Tsitsiklis, J.N.: Problems in Decentralized Decision Making and Computation. In: PhD thesis, Dept. of Electrical Engineering and Computer Science, Massachussetts Institute of Technology (1984)

van Olst, J.C., Hunter, J.R.: Some aspects of the organization of fish schools. J. Fish. Res. Board Can. 27(7), 1225-1238 (1970)

Vicsek, T., Czirók, A., Ben-Jacob, E., Cohen, I., Shochet, O., Tenenbaum, A.: Novel type of phase transition in a system of self-driven particles. Phys. Rev. Lett. 75(6), 1226-1229 (1995)

Weihs, D.: Energetic advantages of burst swimming of fish. J. Theor. Biol. 48(1), 215-229 (1974)

Young, G.F., Scardovi, L., Cavagna, A., Giardina, I., Leonard, N.E.: Starling flock networks manage uncertainty in consensus at low cost. PLoS Comput. Biol. 9(1), e1002894 (2013)

Zhang, F., Leonard, N.E.: Cooperative filters and control for cooperative exploration. IEEE Trans. Autom. Control 55(3), 650-653 (2010) 